\title{
Systematic analysis of somatic mutations driving cancer: uncovering functional protein regions in disease development
}

\author{
Bálint Mészáros ${ }^{1 *}$, András Zeke², Attila Reményi ${ }^{2}$, István Simon ${ }^{1}$ and Zsuzsanna Dosztányi ${ }^{3 *}$
}

\begin{abstract}
Background: Recent advances in sequencing technologies enable the large-scale identification of genes that are affected by various genetic alterations in cancer. However, understanding tumor development requires insights into how these changes cause altered protein function and impaired network regulation in general and/or in specific cancer types.

Results: In this work we present a novel method called iSiMPRe that identifies regions that are significantly enriched in somatic mutations and short in-frame insertions or deletions (indels). Applying this unbiased method to the complete human proteome, by using data enriched through various cancer genome projects, we identified around 500 protein regions which could be linked to one or more of 27 distinct cancer types. These regions covered the majority of known cancer genes, surprisingly even tumor suppressors. Additionally, iSiMPRe also identified novel genes and regions that have not yet been associated with cancer.

Conclusions: While local somatic mutations correspond to only a subset of genetic variations that can lead to cancer, our systematic analyses revealed that they represent an accompanying feature of most cancer driver genes regardless of the primary mechanism by which they are perturbed during tumorigenesis. These results indicate that the accumulation of local somatic mutations can be used to pinpoint genes responsible for cancer formation and can also help to understand the effect of cancer mutations at the level of functional modules in a broad range of cancer driver genes.
\end{abstract}

Reviewers: This article was reviewed by Sándor Pongor, Michael Gromiha and Zoltán Gáspári.

Keywords: Cancer, Driver gene, Somatic mutation, Protein functional modules, Missense mutation, Insertion, Deletion

\section{Background}

Cancer genome projects use next generation sequencing technologies to identify somatic mutations - most often in exonic regions - that discriminate tumor cells from normal cells with the aim to understand the basis of the most common genetic disease [1-5]. The observed genetic alterations showed that the genetic landscape of cancer is complex, affecting a much larger number and

\footnotetext{
*Correspondence: meszaros.balint@ttk.mta.hu; dosztanyi@caesar.elte.hu ${ }^{1}$ Institute of Enzymology, Research Centre for Natural Sciences, Hungarian Academy of Sciences, 2 Magyar Tudósok krt, Budapest H-1117, Hungary ${ }^{3}$ MTA-ELTE Lendület Bioinformatics Research Group, Department of Biochemistry, Eötvös Loránd University, 11/c Pázmány Péter stny, Budapest H-1117, Hungary

Full list of author information is available at the end of the article
}

varied types of genes than previously expected $[1,6]$. There is also heterogeneity at the level of the underlying genetic mechanisms that lead to the variations. With advanced technologies, cancer genome projects are able to produce a more complete catalog of the variations. These include single point mutations and short insertions or deletions that can have a localized effect on a single gene and larger structural aberrations such as copy number alterations and genomic rearrangements that generally affect multiple genes. These data are cataloged in various databases, such as the COSMIC database, which now contains over millions of variations that are dominated by simple mutations [7, 8]. Most of the observed variations, however, correspond to randomly occurring passenger 
mutations. One of the key challenges in the interpretation of cancer genomics data is the identification of driver mutations that provide direct growth advantages in tumorigenesis, and distinguishing them from passenger mutations [3, 4].

Various computational approaches have been developed to identify driver mutations and driver genes in which they reside. Most commonly, individual genes are identified based on the predicted functional impact or the reoccurrence of mutations [3, 9]. In these later approaches, the number of mutations observed for each gene is compared to a background mutation rate. Originally, a uniform background mutation rate was used for the whole genome leading to many falsely identified driver genes. According to recent studies, however, the mutational background rate, which dictates the occurrence of stochastic mutations can also vary at the level of individual genes and depends on genomic location, expression level and replication time [10,11]. The more recent version of the MutSig algorithm incorporates this mutational heterogeneity by using a context dependent estimation of the background mutation rate [10]. This estimation is based on the observed synonymous and intronic mutations for each gene that can be effectively used in tumors with high mutation rates only.

While most efforts traditionally aim at the identification of cancer drivers at the level of genes, often more insight can be gained by taking into account that the position and nature of observed mutations can often be translated to changes of protein function and structure. A fundamental property of proteins is that they can be composed of multiple functional modules that can include a combination of ordered domains and highly flexible intrinsically disordered regions $[12,13]$. These specific regions can have independent functions and produce different phenotypic responses if disrupted. In accordance, it has already been observed that disease causing mutations are often clustered into specific regions of proteins, highlighting the role of specific functional modules in various diseases $[14,15]$.

The concept of identifying specific protein regions which contain a significant number of mutations has been already applied to identify cancer drivers. One method, OncoDriveClust [16] uses coding-silent mutations as background, and detects regions where mutations are clustered compared to this background. However, because of its suboptimal background model, this method often misses even frequently mutated regions, like the DNA binding domain of p53. Using an alternative approach, the e-Driver [17] method relies on predefined functional regions such as known domains or predicted disordered segments. From these segments, the method selects those that show a bias in their mutational rate compared to other functional regions within the same protein. Another type of approach incorporates information about the three-dimensional structure of proteins to evaluate the effect of mutations [18, 19]. Studying the effect of mutations at the level of specific regions gives us a better resolution and might serve as a better tool to understand the molecular principles of various diseases, including cancer $[15,18,20]$. A clear advantage of this approach is that a specific background of mutation frequencies can be calculated for each protein. However, current methods have limitations either because of biases towards known structure or domain assignments or due to an inaccurate background model.

Here we present a novel method called iSiMPRe (identification of significantly mutated protein regions) that is able to pinpoint proteins and protein regions that harbor a significant amount of cancer-related mutations in an unbiased manner. We consider mutations that carry precise and localized information of the affected region of proteins, thus include only missense mutations and inframe insertions and deletions. Significantly mutated regions are identified using a unified statistical model for all three mutation types. Using the annotations of mutations it is also possible to tie significantly mutated protein regions to specific cancer types. We systematically analyzed the performance of iSiMPRe in identifying known cancer genes. We found that a surprisingly complete set of previously established cancer genes can be obtained by using the above mentioned limited set of genetic variations that directly affect protein regions. The main advantage of the new method is that it can pinpoint not only genes but also specific protein regions that are targeted by cancer mutations, even below the level of domains. The analysis of these regions helps to interpret the effect of cancer mutations at the level of functional regions.

\section{Results}

Significantly mutated protein regions are identified based on mutation pattern

In this work we collected cancer associated nonsynonymous mutations from the COSMIC database. We developed a novel method called iSiMPRe that is able to identify cancer genes at the level of protein regions based on these mutations. The underlying assumption of iSiMPRe is that cancer mutations affecting protein coding regions are not distributed evenly, rather they are accumulated in specific regions (Significantly Mutated Protein Regions - SiMPRes) that play an active role in tumorigenesis. Regions that harbor a significantly enriched amount of somatic mutations compared to neutral local mutations can highlight not only cancer genes but also specific functional regions within them that actively contribute to the development of cancer. Since the mutation frequency is calculated for each 
gene separately from the observed number of mutations, different background mutation rates apply for each gene, that can take into account that the background mutation rate is different for individual genes [10]. In our analysis, we only considered exonic mutations that have a local effect, therefore only missense mutations and in-frame insertions and deletions are considered. The presented method is the first that is able to consider in-frame insertions and deletions as well as missense point mutations in a unified statistical framework. This sets it apart from two conceptually similar methods, eDriver [17] and OncoDriveClust [16], which do not consider in-frame insertions and deletions. The input of iSiMPRe is a set of cancer-related missense mutations and in-frame insertions and deletions. The background mutation rate is calculated simply from these using only a few empirical parameters. This is in contrast to OncoDriveClust which estimates the background mutation rate from a set of silent substitutions that has to be supplied as a separate set of input data. iSiMPRe is described in detail in Additional file 1 (iSiMPRe protocol) and in short in Fig. 1. In order to enable potential users to apply the method to updated versions of COSMIC datasets or other sources of cancer mutation data, the source code of iSiMPRe is available for download. In our experience, the identified significantly mutated regions change very little with updates of COSMIC datasets.

The large scale scanning of the COSMIC database yielded a total of 534 SiMPRes in 382 genes in the human proteome. A complete list of identified regions together with their significance levels, the number and type of mutations contained and respective cancer types is shown in Additional file 2. Highly significant mutated regions are usually found in well-established cancer genes such as BRAF and TP53. There are, however, only a limited number of high significance regions as most regions show a more moderate accumulation of mutations with medium to low significance (see Table 1). These results are in agreement with earlier observations that the mutational landscape of cancer is dominated by mutational hills with a few mutational mountains [1]. The length of the identified regions spans from 1 to 280 residues with an average of 20 residues. The identified regions on average cover $3.1 \%$ of a protein's sequence, with only a handful of regions exceeding $35 \%$. These data, indeed, indicate that cancer mutations are not distributed evenly within proteins, but are clustered within certain localized regions.

Known cancer genes harbor significantly mutated regions We have tested the overlap between the identified SiMPRes and the cancer-related genes in the SCGD (see Methods), and separately on annotated tumor suppressors and oncogenes. iSiMPRe can identify at least one SiMPRe in $74 \%$ of the genes in SCGD (Fig. 2). The performance of the method is nearly perfect for oncogenes with at least one identified region (53 out of 54, accounting for $98 \%$ ) with only one exception, DNMT1. In this case, the available mutational data is insufficient to yield high significance. Unlike its relative, DNMT3A, where mutations are highly enriched at a C-terminal region near the catalytic site, studies indicate that mutations of DNMT1 appear to be a rare event [21].

It is typically assumed that oncogenes harbor mainly missense mutations, while tumor suppressor genes are mainly affected by inactivating mutations, most often truncating nonsense or frameshift mutations [22]. As iSiMPRe considers only mutations that are traditionally linked to oncogenes and not to tumor suppressors, the recovery rate calculated on the latter group of genes is expected to be lower compared to oncogenes. In accordance with the hypothesis, a more moderate performance was observed in this case. Nevertheless, the majority of tumor suppressor genes (50 out of 71, accounting for $70 \%$ ) were still identified as containing at least one region with a significant accumulation of local somatic mutations. This is quite a striking result given that the contribution of missense mutations to tumor suppressor gene alterations is considered to be secondary to inactivating and truncating mutations [22].

\section{iSiMPRe outperforms already existing methods with the same scope}

Additionally to iSiMPRe, two recently developed methods OncoDriveClust [16] and eDriver [17] were also tested. These methods - similarly to our approach - aim to identify regions where mutations are clustered in the amino acid sequence. The major difference between eDriver and iSiMPRe is that eDriver relies on regions with predefined boundaries based on domain annotations and disorder predictions, while iSiMPRe identifies regions that maximize mutational significance without relying on predefined regions. While the background model of eDriver is similar to the one used in our model, OncoDriveClust uses different input data that includes frameshift and nonsense mutations together with missense point mutations and compares it to the distribution of silence mutations.

Figure 2a shows the number of genes identified by the three methods together with the overlap between them. The overlap between iSiMPRe and eDriver is high with most eDriver genes and regions being identified by iSiMPRe as well. However, the overlap between genes identified by iSiMPRe and OncoDriveClust was significantly lower. In terms of performance a similar trend was followed for the three methods (Fig. 2b-d). Oncogenes were recognized best, while tumor suppressors are 
Collect local somatic mutations from cosmic

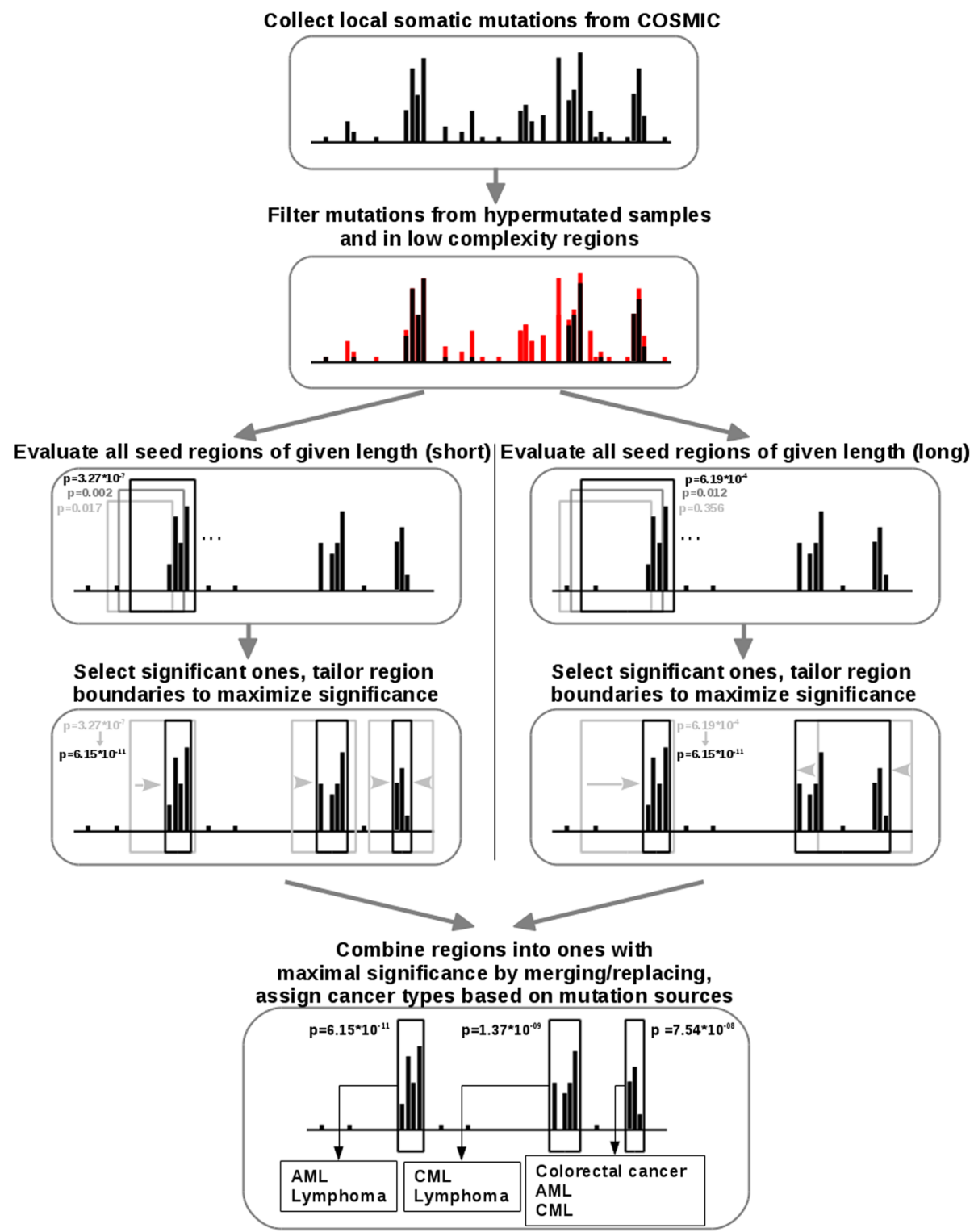

Fig. 1 Outline of the method. All local somatic mutations are collected from the COSMIC database for a given gene, discarding mutations coming from hypermutated samples (see Methods) and mutations overlapping with low complexity regions. Next, a seed region in the corresponding protein sequence is selected and is assessed for significant enrichment of mutations compared to the expected random distribution using a one-sided Fisher's exact test. Next, if the selected region is significant ( $p$-value $<0.01)$ its boundaries are moved to either side to locally maximize significance. This is repeated for all possible seed regions of 7, 10 and 30 residues in length. After the evaluation of all seed regions, the resulting optimized regions are merged if overlap occurs between them. For an exhaustive description of the algorithm see Additional file 1

more challenging, especially for OncoDriveClust. In all three datasets, iSiMPRe achieves the best performance, followed by eDriver. The lower performance of concurrent methods can be traced back to various factors. In the case of eDriver, averaging mutation numbers in larger predefined regions can sometimes mask significant regions, which can have a negative effect on correctly identifying cancer driving genes. Trivially, no significantly mutated regions could appear in proteins composed of a single domain or one disordered region alone. The performance of OncoDriveClust was significantly lower, with only one tumor suppressor and only less than third of oncogenes identified. This lower performance of OncoDriveClust suggests that its expected mutation distribution statistical model should be revisited and that the larger scale effect of frameshift/ 
Table 1 Summary of identified SiMPRes. Regions are grouped according to their significance level (see Methods) and their dominant mutation type

\begin{tabular}{|c|c|c|c|c|c|}
\hline & & \multicolumn{4}{|c|}{ Dominant mutation type } \\
\hline & & Missense mutations & Insertions & Deletions & Total \\
\hline \multirow[t]{4}{*}{ Significance level } & High significance & 68 & 5 & 10 & $83(15.5 \%)$ \\
\hline & Medium significance & 77 & 0 & 13 & $90(16.9 \%)$ \\
\hline & Low significance & 285 & 9 & 67 & $361(67.6 \%)$ \\
\hline & TOTAL & $430(80.5 \%)$ & $14(2.6 \%)$ & $90(16.9 \%)$ & $534(100 \%)$ \\
\hline
\end{tabular}

nonsense mutations cannot be compared to the local effect of missense mutations and short indels.

\section{In-frame indels are important for finding cancer genes}

The used version of the COSMIC database contains 404,903 missense mutations and 9977 in-frame insertions and deletions. While the number of short insertions and deletions is only less than $2.5 \%$ of that of all local somatic mutations, they still make significant contribution to the identification of significantly mutated regions and contribute to the increased performance of iSiMPRe. These types of genetic alterations are dominant in about $20 \%$ of the found regions, meaning indels in these cases contribute more to the significance of the region than missense mutations. In accordance, one-fifth of all regions are undetectable without taking indels into account. Omitting indels from the calculations would result in a decreased performance. Nevertheless, iSiMPRe would still outperform the other two methods (Fig. 2b-d). The incorporation of indel type mutations is especially important for the identification of tumor suppressors. Omitting indels does not have an impact on the recognition of oncogenes, but it does affect the results on both SCGD and tumor suppressors.

Some of the significantly mutated indel regions occur within genes with a well-established connection with cancer development, such as KIT, BRAF or PTEN. The proteins encoded by these genes harbor multiple SiMPRes,

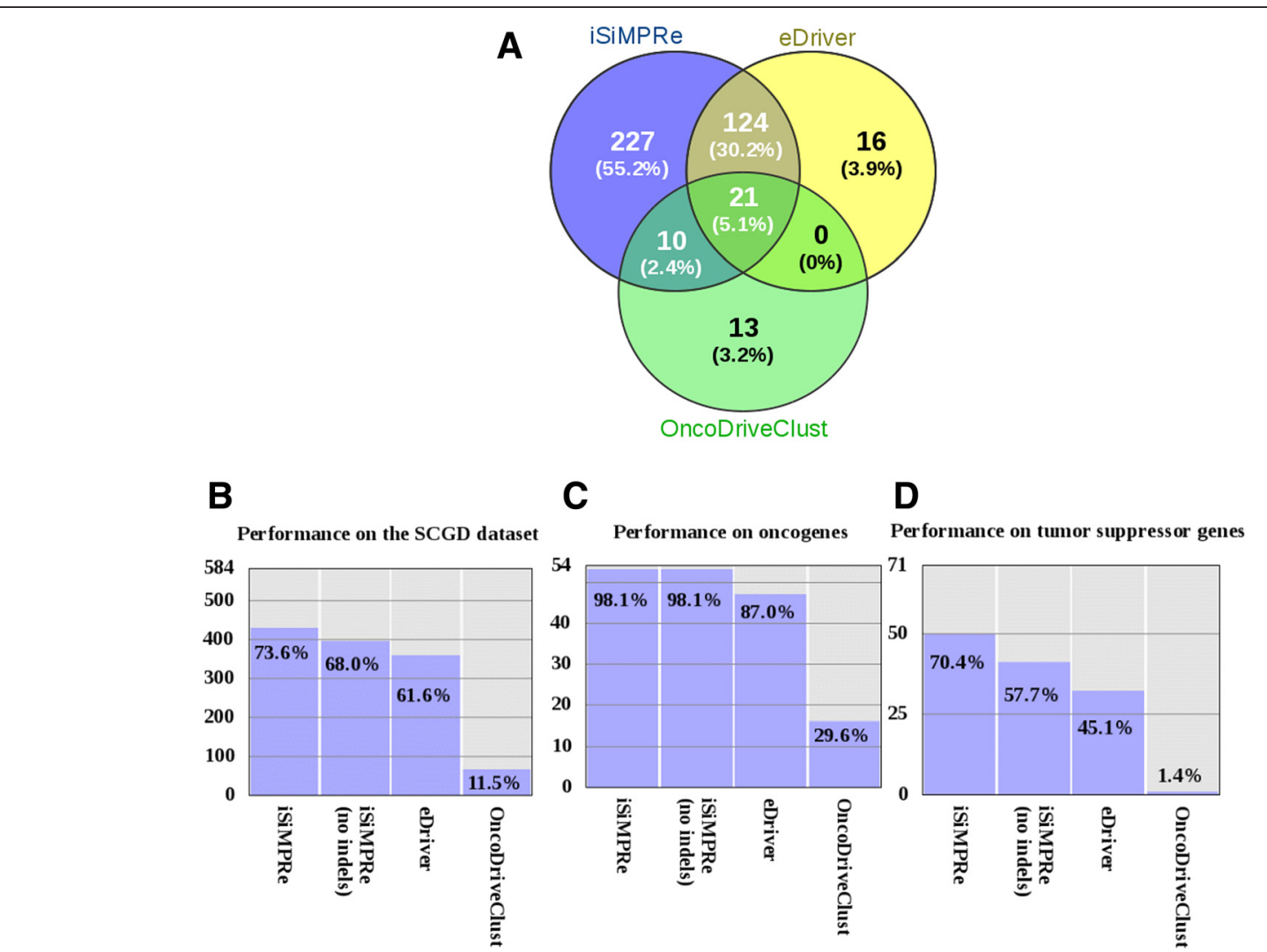

Fig. 2 Comparison of the effectiveness and overlap between methods. Number of genes (a) identified by the different methods together with respective overlaps. The recovery rates of all three methods tested on the SCGD (b), oncogenes (c) and tumor suppressors (d) 
many of which are dominated by missense mutations, making the genes themselves identifiable using missense mutations alone. However, in certain cancer genes the primary mechanism of genetic alteration is the accumulation of short insertions or deletions. Discarding indels in the region identification process would make it impossible to reliably identify such known cancer genes. Notable examples include the interleukin-7 receptor subunit alpha (IL7R), which is known to be involved in the occurrence and development of various forms of acute leukemias and solid tumors [23]. Other examples include TSC2, where mutations have been linked to the development of hamartomas in multiple organs [24]; and ATAD5, the somatic mutations of which were identified in endometrial tumors [25].

\section{Other types of genetic alterations are also often associated with local somatic mutations}

Known cancer genes are defined based on various dominant type of genetic alterations and do not necessarily contain an accumulation of local somatic mutations. In order to gain insights into how the dominant type of genetic alteration for a given gene influences the chance of iSiMPRe to find a significantly mutated region at the protein level, the performance was further analyzed on specific datasets. For this analysis the KEGG database was used that provides information about a broad set of genetic alterations associated with specific genes. Figure 3 shows the ratio of genes for each type of dominant genetic alteration where SiMPRes were identified in the protein counterparts. Ten categories were analyzed in which somatic mutations represent only one of the categories. The results indicate that chromosomal translocations and rearrangements represent a roughly independent modulation of the genome and genes primarily subject to such changes are largely devoid of significantly mutated regions. Also, relatively modest recovery rate was observed for genes altered dominantly via germline mutations. In general, more than $80 \%$ of the proteins dominantly altered by other mechanisms also contain at least one significant region identified by iSiMPRe in seven out of ten categories.

\section{Significantly mutated regions can be specific to one cancer type or can be associated with a broad set of cancer types}

Based on the supplied annotations in the respective datasets, genes were linked to one or more of the 27 standardized cancer types (see Methods and Table 2). Taking advantage of this annotation, the performance of iSiMPRe could be evaluated for individual cancer types. Figure 4 indicates that the overlap between cancer genes and significantly mutated regions found by iSiMPRe is highly uneven across various cancer types. In certain cancer types, such as head and neck carcinomas, iSiMPRe is able to identify all known cancer genes, exhibiting a perfect performance. In other cases the recovery rate is lower (such as the $40 \%$ in the case of prostate

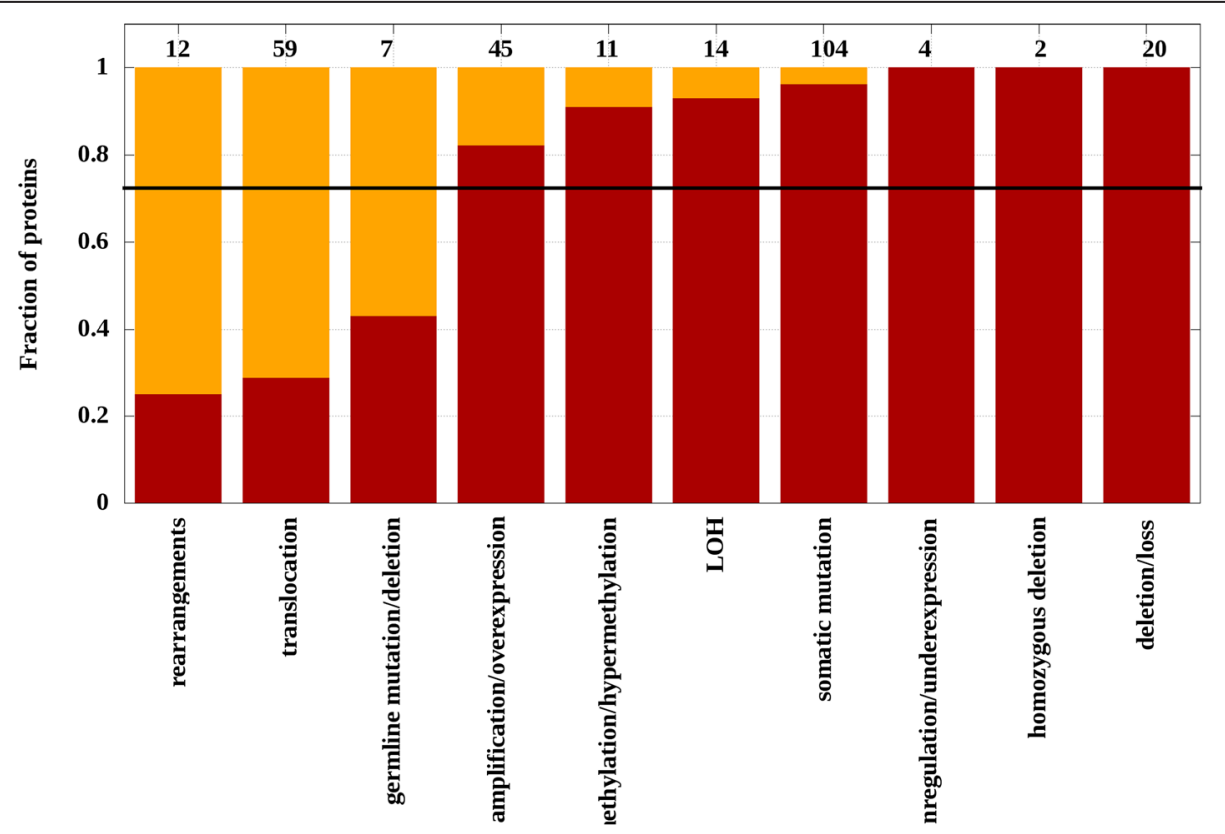

Fig. 3 The ratio of proteins with significantly mutated regions in various classes of typical genetic alterations. Based on the KEGG annotation, genes are grouped according to the dominant genetic aberrations for a given gene. Red bars show the fraction of proteins with found regions, the horizontal black line shows the average ratio of 0.752 . Numbers above bars show the number of known genes in the category. (LOH $=\mathrm{LOsS}$ of heterozygosity) 
Table 2 Twenty-seven main cancer types. Cancer types are shown with the corresponding tissue/organ of occurrence and number of local somatic mutations and originating samples in COSMIC

\begin{tabular}{|c|c|c|c|c|}
\hline Tissue/organ & Cancer types & $\begin{array}{l}\text { Number of local somatic } \\
\text { mutations in COSMIC }\end{array}$ & Number of samples & $\begin{array}{l}\text { Average number of } \\
\text { mutations per sample }\end{array}$ \\
\hline Bladder & Bladder cancer & 6265 & 3027 & 2.07 \\
\hline \multirow[t]{3}{*}{ Blood } & Acute myeloid leukemia & 11650 & 5857 & 1.99 \\
\hline & Chronic myeloid leukemia & 1603 & 1041 & 1.54 \\
\hline & Lymphoma & 24572 & 6944 & 3.54 \\
\hline Bone & Bone cancer & 2814 & 706 & 3.99 \\
\hline \multirow[t]{4}{*}{ Brain } & Glioblastoma & 9361 & 2909 & 3.22 \\
\hline & Neuroblastoma & 4932 & 619 & 7.97 \\
\hline & Glioma & 12793 & 2947 & 4.34 \\
\hline & Medulloblastoma & 4701 & 728 & 6.46 \\
\hline Breast & Breast cancer & 31544 & 4404 & 7.16 \\
\hline Cervix & Cervical cancer & 136 & 132 & 1.03 \\
\hline Colorectal & Colorectal cancer & 37727 & 25806 & 1.46 \\
\hline Esophagus & Esophageal cancer & 3659 & 433 & 8.45 \\
\hline \multirow[t]{2}{*}{ Head and neck } & Thyroid cancer & 19975 & 13908 & 1.44 \\
\hline & Head and neck carcinoma & 75 & 67 & 1.12 \\
\hline Kidney & Renal cell carcinoma & 27897 & 1750 & 15.94 \\
\hline Liver & Hepatocellular carcinoma & 19100 & 2091 & 9.13 \\
\hline \multirow[t]{2}{*}{ Lung } & Small cell lung cancer & 976 & 208 & 4.69 \\
\hline & Non-small cell lung cancer & 19991 & 11236 & 1.78 \\
\hline Ovary & Ovarian cancer & 19286 & 2936 & 6.57 \\
\hline Pancreas & Pancreatic cancer & 33776 & 5609 & 6.02 \\
\hline Prostate & Prostate cancer & 18813 & 967 & 19.46 \\
\hline \multirow[t]{3}{*}{ Skin } & Melanoma & 12374 & 7923 & 1.56 \\
\hline & Squamous cell carcinoma & 15726 & 3459 & 4.55 \\
\hline & Basal cell carcinoma & 292 & 251 & 1.16 \\
\hline Stomach & Stomach cancer & 7795 & 1430 & 5.45 \\
\hline Uterus & Endometrial cancer & 5722 & 1627 & 3.52 \\
\hline Total & & 353555 & 109015 & 3.24 \\
\hline
\end{tabular}

and bone cancer). However, iSiMPRe is able to recover $74 \%$ of cancer genes on average with recovering at least one third of genes even in the poorest case. Although perfect performance can be seen in some cancer types with low numbers of COSMIC mutations (such as stomach cancer, head and neck carcinoma, basal cell carcinoma and cervical cancer); in general there is no obvious relationship between the recovery rates and cancer types, sample number or the number of mutations.

At the level of individual genes, cancer genes can be specific to one or a very limited number of (usually related) forms of cancer or can be involved in multiple cancer types. In a number of cases, multiple regions showed a significant enrichment of local mutations within a single gene. In these cases two basic scenarios could emerge. In the first scenario, mutations from various tissue samples are typically distributed in roughly the same way and their accumulation along the sequence outline the same functional regions. This general trend is demonstrated in Fig. 5a in the case of DNA methyltransferase 3A (DNMT3A) and for phosphatidylinositol 3-kinase regulatory subunit alpha (PIK3-R1). In the case of DNMT3A, the dominant part of mutations come from various hematopoietic cancers, such as acute myeloid leukemia (AML), chronic myeloid leukemia (CML) or lymphoma. These mutations cluster in three distinct regions: one region falls into the middle of the ADD (ATRX-DNMT3-DNMT3L) domain, responsible for the interaction with the polycomb repressive complex 2 (PRC2), and it roughly covers the PHD-type zinc finger; the second and the third regions both fall into the catalytic (DNA cytosine 


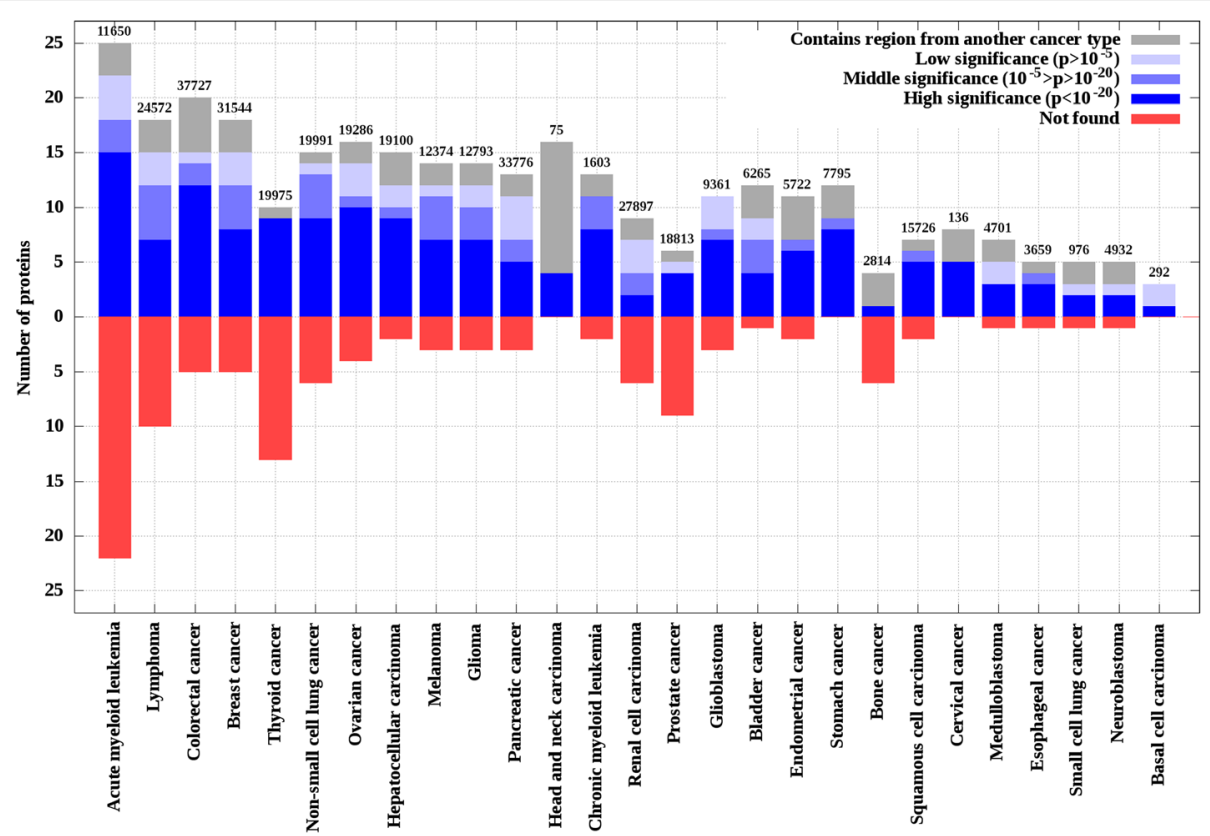

Fig. 4 Overlap between cancer-related genes and significantly mutated regions. Blue bars show proteins that harbor at least one region annotated to the correct cancer-type. Shades of blue show the significance level of the most significant region found. Grey bars show the number of proteins that harbor at least one region but where the region is annotated to a different cancer type. Red bars show the number of proteins without significant regions. Numbers above the bars show the number of mutations in the COSMIC database annotated to the cancer type. Order of cancer types reflects the decreasing number of known genes from left to right

methyltransferase) domain. In all three regions, the source of these mutations is fairly homogeneous with respect to the originating cancer types, even though the dominant mutation types differ for the three regions (deletions + insertions, deletions + missense and missense only, respectively). Similarly, the mutations in PI3K-R1 outline two distinct regions, covering the two terminal parts of the inter-SH2 coiled-coil region, which is responsible for the interaction with the catalytic subunit. Similarly to the DNMT3A case, the regions differ slightly in their dominant mutation types; however, in all regions the majority of the mutations come from the same, endometrial and breast cancer, samples.

Less frequently, a different scenario can also be observed, where a single gene is associated with different types of cancer depending on the location of the mutations. The most interesting examples involve certain tyrosine kinase receptors. Figure $5 \mathrm{~b}$ shows the domain structure and the significantly mutated regions encountered in c-KIT and FGFR3. Both are single-pass transmembrane proteins: in their extracellular region they contain multiple Ig-like domains, while their intracellular regions harbor a tyrosine kinase domain. The latter element is one of the most frequently mutated domains in various cancers (Table 3 ) and it also contains significantly mutated regions. However, other regions in these two proteins also exhibit an increased number of genetic variations that can involve mostly missense mutations, deletions, insertions or mixed types of variations. Importantly, these specific regions are associated with different cancer types. In the case of KIT, the majority of known somatic mutations were sequenced in AML, melanoma and gastrointestinal stromal tumor (GIST) samples. The two regions found in the last Ig-like domain of the extracellular receptor part is linked to AML and GIST, respectively. The intracellular regions are either GIST, AML or melanoma specific, or a combination of two of the three cancer types, but interestingly KIT does not seem to harbor a ubiquitously mutated region. Another uncommon cancer-type specific partition of mutations can be seen in the case of FGFR3, where both extracellular regions are linked exclusively to bladder cancer while the intracellular regions are present in various forms of skin tumors as well.

\section{Significantly mutated protein regions correspond to a diverse set of functional modules}

The identified SiMPRes enable us to take a deeper look into which functional modules of proteins are involved in cancer development. For this, we collected biological annotations of the identified regions/genes. Information was collected from UniProt database [26] which is a rich source of information about active sites of enzymes and additional functional sites including nucleotide phosphate-, DNA- and other binding regions. Another annotation category corresponds to 'regions of interest'. This category used by UniProt can refer to basically any 

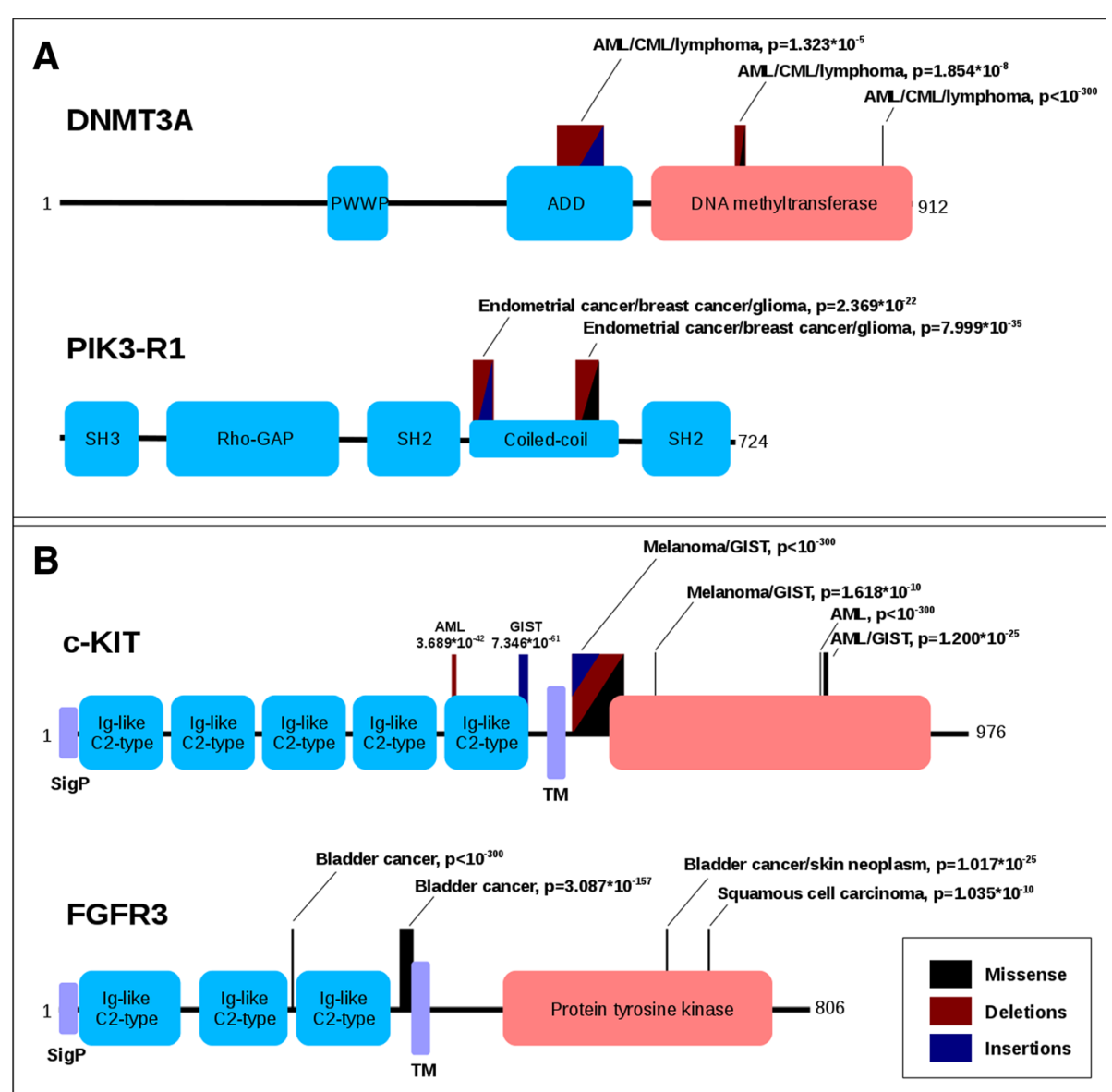

Fig. 5 Proteins with multiple regions covering multiple cancer types. Symmetrically positioned boxes represent structural/functional protein units: grey - signal sequence, black - transmembrane region, various colors - domains (red=catalytic domains, blue =all other domains, with abbreviated names written in the box). Boxes above the line represent significantly mutated regions. Colors denote dominant mutation types: black - missense, red - deletions, blue - insertions. Regions are flagged with dominant cancer types together with the $p$-value of the region. GIST - Gastrointestinal stromal tumor, AML - Acute Myeloid Leukemia, CML - Chronic Myeloid Leukemia. All examples feature multiple regions involved in the same cancer types (a) or multiple cancer-specific regions (b)

experimentally characterized protein region that can be of interest concerning protein function (such as interaction sites, different regions of multifunctional enzymes or regions crucial for biological processes/sub-cellular localizations). UniProt annotations were complemented with annotations from the Pfam database [27] collecting evolutionarily conserved protein family information with associated functions and from the ELM database which provides information about linear motifs operating largely outside of globular domains [28]. The occurrence of SiMPRes in various types of annotations were tested for over and under-representation using a basic statistical model (see Methods).

In most cases the identified SiMPRes could be associated with some structural or functional annotations. The mean overlaps and standard errors of randomized regions is shown in Table 3 together with the overlaps of real SiMPRes. The data in Table 3 shows that local genetic alterations are significantly concentrated on the structured parts of proteins. This is in accordance with earlier findings [29]. Within structured domains, there is a clear tendency for enzymes, especially for kinases, confirming earlier observations [30, 31]. The most commonly mutated domain is the Tyrosine kinase domain observed in 20 different proteins, including such wellknown cancer driver genes as ALK, EGFR or BRAF (see Additional file 3). Other domains mutated in multiple cases include the Serine/threonine-protein kinase domain (e.g. in Activin receptor type-1 or in Serine/threonineprotein kinase STK11) and the SH2 domains (e.g. in PIK3-R1 and STAT3). In theory, the easiest way to disrupt enzyme function would be the modulation of the active site via the introduction of mutations. However, data show that the number of active sites overlapping with SiMPRes is much lower than the number of targeted enzyme/kinase domains. Instead, iSiMPRe showed 
Table 3 The occurrence of SiMPRes in known structural/functional protein sites/regions. Color codes represent over- and underrepresentation compared to random. Shades of red show increasing over-representation. The amount of over- and underrepresentation is given in standard deviation units calculated from 1000 randomly assigned regions. 'Regions of interest' marks experimentally characterized protein regions that can be of interest concerning protein function (such as interaction sites, different regions of multifunctional enzymes or regions crucial for biological processes/sub-cellular localizations)

\begin{tabular}{|c|c|c|c|c|c|}
\hline & & $\begin{array}{l}\text { Number of } \\
\text { regions }\end{array}$ & $\begin{array}{c}\text { Average } \\
\text { from } \\
\text { random }\end{array}$ & $\begin{array}{c}\text { StDev } \\
\text { from } \\
\text { random }\end{array}$ & $\begin{array}{l}\text { Over/under- } \\
\text { representation }\end{array}$ \\
\hline \multirow{5}{*}{ Pfam } & Domains & 199 & 133 & 10.3 & 6.41 \\
\hline & Enzymes & 75 & 25.2 & 5.0 & 9.96 \\
\hline & Kinases & 58 & 9.0 & 3.0 & 16.33 \\
\hline & Motifs & 4 & 0.64 & 0.87 & 3.86 \\
\hline & Repeats & 12 & 13.4 & 4.2 & -0.33 \\
\hline \multirow{7}{*}{ UniProt } & DNA binding regions & 8 & 2.6 & 1.9 & 2.84 \\
\hline & Regions of interest & 113 & 38.2 & 8.6 & 8.70 \\
\hline & $\begin{array}{c}\text { Transmembrane } \\
\text { regions }\end{array}$ & 30 & 27.8 & 6.5 & 0.34 \\
\hline & $\begin{array}{c}\text { Nucleotide phosphate } \\
\text { binding regions }\end{array}$ & 25 & 3.1 & 2.2 & 9.95 \\
\hline & Active sites & 8 & 2.0 & 1.5 & 4.07 \\
\hline & Binding sites & 32 & 2.7 & 2.2 & 13.32 \\
\hline & $\begin{array}{l}\text { Post-translational } \\
\text { modification sites }\end{array}$ & 97 & 26.6 & 8.9 & 7.91 \\
\hline $\begin{array}{c}\text { phospho } \\
\text { ELM }\end{array}$ & Phosphorylation sites & 114 & 29 & 14.1 & 6.05 \\
\hline \multirow{2}{*}{ ELM } & Motifs & 10 & 1.0 & 1.3 & 6.77 \\
\hline & Switches & 16 & 1.0 & 2.1 & 7.14 \\
\hline
\end{tabular}

a highly significant overlap with other types of functional regions, including nucleotide phosphate-, DNA- and other binding regions, as well as 'regions of interest'. Protein binding regions were suggested to be the primary hotspots for tumor suppressor proteins [15]. The high accumulation of mutations was not restricted to structured protein modules. The non-globular parts of proteins - especially motifs, motif switches and post-translational modification sites - tended to coincide with a high accumulation of mutations as well $[32,33]$.

\section{Discussion}

\section{The search for cancer driver genes continues}

Cancer emerges due to genetic and epigenetic changes. With the dramatic expansion of catalogued mutations detected in diverse tumor samples and the advent of exome sequencing screens, we have an overwhelming amount of data on our hands. Although this - in theory should enable easier insights into tumorigenesis, it is still not a simple task to adequately distinguish between relevant, cancer-associated mutations and background genetic alterations without clinical significance. Therefore, one of the major focuses of cancer research is still the identification of genes that are responsible for cancer formation, tumor progression and metastasis. However, the definition of these cancer genes is far from trivial. Usually a prerequisite of a cancer driver gene is that its genetic alterations should have a positive contribution to the development of cancer. In practice, the decision of whether a gene fulfills these criteria or not can depend on the cancer type and the number of samples already analyzed, as well as the type of genetic alterations analyzed. It was also suggested that significantly larger sample size is needed to obtain a complete set of cancer driver genes [34]. The subjectivity of cancer drivers is transferred to the publicly available databases in which many such genes are collected. These datasets use different levels of evidence for the inclusion of a gene or gene product and they can also contain erroneously identified cancer genes, for example based on mouse models or paralog sequences. Since currently there is no consensus on the list of cancer genes, we used four different sources to collect and combine manually assembled and curated sets of cancer genes (see Methods and the figure in Additional file 4).

The lack of consensus regarding cancer genes is evident from the limited overlap between the four included datasets. The fact that only around $27 \%$ of OMIM genes have support in other databases can be attributed to the fact that OMIM is primarily aimed at collecting germline affected genes that only partially overlap with somatically altered genes. Unexpectedly, even more limited overlap was observed in the case of COSMIC census database. For this dataset over half of the listed genes were absent from all other databases, as opposed to the $20 \%$ and $4 \%$ of the genes listed in KEGG and Driver genes, respectively. It could mean that the COSMIC census list includes novel cancer genes that are not present in other datasets as of yet, or it could also indicate lack of supportive evidence and possible biases of the inclusion system.

In addition to manually curated databases, various computational methods have also been developed to identify cancer driver genes [35, 36]. Most commonly used approaches seek to identify cancer driver genes either in the context of pathways and protein interaction networks [37] or by detecting signs of positive selection at the level of genes. Such methods can be based on the 
increased number of observed mutations compared to the background mutation rate [10]; a high rate of nonsilent mutations compared to silent mutations [2]; or on the bias towards the accumulation of mutations with high functional impact [38]. However, the accumulation of mutations can highlight not only genes but also specific functional regions at the protein level that are involved in disease development. Recent methods, such as OncoDriveClust [16] or e-Driver [17] identify putative cancer driver genes based on this concept, similarly to the iSiMPre method presented in this work. Clustering of mutations can also be observed in three-dimensional protein structures that often correspond to perturbed protein-protein interaction sites $[18,19]$. Given the complexity and heterogeneity of the molecular basis of cancer, the combination of different signals of positive selection can more reliably indicate mutational drivers [39]. Nevertheless, in our experience, the iSiMPre method is able to identify the majority of cancer driver genes based on the clustering of mutations and outperforms methods with similar scope. The increased performance of iSiMPre can be attributed to several factors, including the cleaning of mutational data (e.g. eliminating likely neutral polymorphisms and mutations occurring within tandem repeats that are more likely to accommodate neutral mutations as well as sequencing errors). Additional factor is the incorporation of all genetic variations with positional information, which include short in-frame insertions and deletions while excluding frameshift and non-sense mutations. iSiMPre is based on an unbiased approach that does not rely on previous knowledge of structure or domain, which could be especially important to detect cancer driver mutations located in intrinsically disordered proteins for example (manuscript in preparation).

Based on the presented analysis, the local accumulation of somatic mutations detected by iSiMPRe can also be used to pinpoint novel genes not yet (fully) represented in the available databases. We found 7 genes that contained high significance mutated regions that were absent from SCGD. In these cases, the high significance of the enrichment of somatic mutations lends a very strong support that these proteins indeed correspond to true cancer drivers. These include the lymphoma gene IL7R, the $\mathrm{K}^{+}$channel KCNJ5, a hyperaldosteronismlinked gene that is also known to be mutated in adrenal cancers [40], CD79B known to be involved in B-cell lymphomas [41], the breast cancer gene ESR1 [42], the cytokine receptor IL6ST known to be involved in a range of cancers, the known cancer gene RAC1 regulating cell motility and RHOA also involved in various cancers, eg. gastric carcinomas [43]. For these 7 genes that are unique to the COSMIC census dataset, iSiMPRe confirms their cancer driver statuses.
Moving towards lower significance levels, the list of novel cancer genes becomes more populated. Altogether, 23 novel genes are identified by iSiMPRe with medium significance level, 18 of which are included in only one dataset. In these cases, the presence of a reliable region and the inclusion in one cancer gene list is a very strong indication of being a true cancer gene. This notion is supported by genes such as the checkpoint kinase 2 (CHEK2) and tuberin, which have been described as tumor suppressors; or CSF3R which has been described as an oncogene [44]. The other 15 genes harboring medium significance regions are even less represented in cancer gene datasets as they are absent from all four studied databases. These genes are shown in Table 4. Although missing from the studied databases, there is at least some indication of the genes' involvement in cancer in the majority of cases. For RP1L1, recent results might offer a link between mutations in this gene and the development of gastric and colorectal cancers [45]. Similarly, mucin 6 is known to be linked to various forms of cancer [46, 47]. For other genes, their involvement in cancer is only hinted at in very preliminary studies (FRG1B) [25, 48, 49]. In addition, a link between WASH3P and cancer is supported by our findings; since mutations in a region indicate involvement in NSCLC and renal cell carcinoma.

iSiMPRe also identified 211 low significance regions that reside in genes not present in any of the cancer gene datasets (see table in Additional file 2). Although some of these genes have recently been linked to tumorigenesis (eg. FRK was shown to be involved in hepatocellular adenomas, which matches our annotations [50]), most genes have no direct indication of being cancer genes. Some of these candidate genes might be linked causatively to tumorigenesis, but they can only be expected to exhibit a weak phenotype (small growth advantage versus wild-type cells) in agreement with their lower mutation hit rate. However, they can also correspond to genes with a locally increased passenger mutation rate, without true biological significance. In these cases more data is needed to be able to discriminate low significance regions that might correspond to false positives from those regions that are genuinely involved in various cancers even though just with a weak phenotype.

Our results indicate that iSiMPRe is able not only to identify known and novel cancer genes, but the assigned confidence levels of the identified regions correlate well with our current knowledge of cancer driver genes. Furtermore, iSiMPRe can also effectively target the main issue of interpreting genomic sequencing data: the discrimination of driver and passenger mutations. Overall, the regions marked by iSiMPRe only contain about one third of the mutations listed in COSMIC, which is a drastic reduction of data. As there is a significant 
Table 4 Medium significance region genes that are absent from all somatic cancer gene databases

\begin{tabular}{|c|c|c|c|c|c|c|c|}
\hline Gene & Region & $p$-value & $\begin{array}{l}\text { Dominant } \\
\text { cancer type(s) }\end{array}$ & Protein name & Protein annotations & Region annotations & $\begin{array}{l}\text { Indication of involvement in } \\
\text { cancer }\end{array}$ \\
\hline WASH3P & $368-410$ & $1.050^{*} 10^{-14}$ & $\begin{array}{l}\text { Renal cell } \\
\text { carcinoma }\end{array}$ & $\begin{array}{l}\text { Putative WAS protein } \\
\text { family homolog } 3\end{array}$ & $\begin{array}{l}\text { Pseudogene homolog of WASP, } \\
\text { nucleation-promoting factor of } \\
\text { endosomes }\end{array}$ & $\begin{array}{l}\text { Missense mutations affect mainly } \\
\text { one position. Region is part of } \\
\text { Pfam-B conserved accross wide } \\
\text { range of eukaryotes and probably } \\
\text { disordered }\end{array}$ & $\begin{array}{l}\text { Some indication of possible } \\
\text { involvement in tumors (PMID: } \\
\text { 21208217) }\end{array}$ \\
\hline FRG1B/C20orf80 & 40-101 & $7.492^{*} 10^{-14}$ & $\begin{array}{l}\text { Prostate cancer, } \\
\text { Glioma }\end{array}$ & Protein FRG1B & Unknown & $\begin{array}{l}\text { Well distributed missense mutations } \\
\text { in structured FRG1 domain, no } \\
\text { known function, but conserved } \\
\text { across eukar + some bact. }\end{array}$ & $\begin{array}{l}\text { Only based on mutation pattern, } \\
\text { no cancer specific annotations }\end{array}$ \\
\hline $\begin{array}{l}\text { ANKRD36C/ } \\
\text { ENSG00000174501 }\end{array}$ & $626-634$ & $2.620 * 10^{-12}$ & $\begin{array}{l}\text { Prostate cancer, } \\
\text { Glioma }\end{array}$ & $\begin{array}{l}\text { Ankyrin repeat } \\
\text { domain- containing } \\
\text { protein } 36 C\end{array}$ & Unknown & $\begin{array}{l}\text { Well clustered missense mutation } \\
\text { peaks in an unannotated, possibly } \\
\text { disordered region of the protein }\end{array}$ & $\begin{array}{l}\text { Very pleriminaty indication } \\
\text { of possible role in various } \\
\text { cancer types }\end{array}$ \\
\hline ZNF814 & $337-337$ & $6.451 * 10^{-11}$ & $\begin{array}{l}\text { Pancreatic } \\
\text { cancer, } \\
\text { Squamous cell } \\
\text { carcinoma }\end{array}$ & $\begin{array}{l}\text { Putative } \\
\text { uncharacterized zinc } \\
\text { finger protein } 814\end{array}$ & $\begin{array}{l}\text { Acts as a trascription factor with } \\
\text { specific DNA binding }\end{array}$ & $\begin{array}{l}\text { Sharp peak of missense mutations } \\
\text { N-terminal of the zinc binding } \\
\text { domains }\end{array}$ & $\begin{array}{l}\text { Very pleriminaty indication of } \\
\text { possible role in some cancer } \\
\text { types }\end{array}$ \\
\hline RP1L1 & $1305-1361$ & $1.654^{*} 10^{-9}$ & Various & $\begin{array}{l}\text { Retinitis pigmentosa } \\
1 \text {-like } 1 \text { protein }\end{array}$ & $\begin{array}{l}\text { Involved in axoneme assembly, } \\
\text { photoreceptor cell development } \\
\text { and retina development in } \\
\text { camera-type eye }\end{array}$ & $\begin{array}{l}\text { Broad peak of missense mutations } \\
\text { and indels in the central, possibly } \\
\text { disordered region of the protein }\end{array}$ & $\begin{array}{l}\text { Indication of involvement in } \\
\text { gastric and colorectar cancers } \\
\text { (PMID: 23237666) }\end{array}$ \\
\hline $\begin{array}{l}\text { RRN3P2/ } \\
\text { ENSG00000103472 }\end{array}$ & $368-375$ & $1.676^{*} 10^{-9}$ & Prostate cancer & $\begin{array}{l}\text { RRN3 homolog, RNA } \\
\text { polymerase I } \\
\text { transcription factor } \\
\text { pseudogene } 2\end{array}$ & Unknown & $\begin{array}{l}\text { Sharp peak of missense mutations } \\
\text { in the RRN3 domain }\end{array}$ & Unknown \\
\hline MUC6 & 1873-1995 & $3.663^{*} 10^{-7}$ & Prostate cancer & Mucin 6 & $\begin{array}{l}\text { Modulates the composition of the } \\
\text { protective mucus layer. Important } \\
\text { in the cytoprotection of pithelial } \\
\text { surfaces, used as tumor markers } \\
\text { in a variety of cancers. May play } \\
\text { a role in epithelial organogenesis. }\end{array}$ & $\begin{array}{l}\text { Broad peak of missense mutations } \\
\text { in a possibly disordered region of } \\
\text { the protein }\end{array}$ & $\begin{array}{l}\text { Known to be linked various forms } \\
\text { of cancer (PMID: 21851820, } \\
\text { PMID: 9650551) }\end{array}$ \\
\hline EEF1B2 & $43-43$ & $3.739 * 10^{-7}$ & Prostate cancer & $\begin{array}{l}\text { Elongation factor } \\
\text { 1-beta }\end{array}$ & $\begin{array}{l}\text { Translation elongation factor, guanine } \\
\text { nucleotide exchange factor involved } \\
\text { in the transfer of aminoacylated } \\
\text { tRNAs to the ribosome }\end{array}$ & $\begin{array}{l}\text { Sharp peak of missense mutations } \\
\text { in the N-terminal region of the } \\
\text { protein }\end{array}$ & Unknown \\
\hline POTEC & $477-511$ & $4.504^{*} 10^{-7}$ & Prostate cancer & $\begin{array}{l}\text { POTE ankyrin domain } \\
\text { family member } C\end{array}$ & Unknown & $\begin{array}{l}\text { Multiple peaks of missense } \\
\text { mutations in the C-terminal } \\
\text { disordered part of the protein, } \\
\text { encompassing a possible DNA } \\
\text { binding motif }\end{array}$ & Unknown \\
\hline EIF1AX & $2-15$ & $1.457^{*} 10^{-6}$ & $\begin{array}{l}\text { Thyroid cancer, } \\
\text { Melanoma }\end{array}$ & $\begin{array}{l}\text { Eukaryotic translation } \\
\text { initiation factor } 1 \mathrm{~A} \\
\text { X-chromosomal }\end{array}$ & $\begin{array}{l}\text { Required for maximal rate of protein } \\
\text { biosynthesis, enhances ribosome } \\
\text { dissociation }\end{array}$ & $\begin{array}{l}\mathrm{N} \text {-terminal disordered region, } \\
\text { harboring many missense mutations }\end{array}$ & $\begin{array}{l}\text { Indication of involvement in } \\
\text { melanoma (PMID: 24423917) }\end{array}$ \\
\hline CS & 183-187 & $1.670 * 10^{-6}$ & & $\begin{array}{l}\text { Mitochondrial citrate } \\
\text { synthase }\end{array}$ & & & $\begin{array}{l}\text { Indication of involvement in } \\
\text { some cancers (PMID: 19647716) }\end{array}$ \\
\hline
\end{tabular}


Table 4 Medium significance region genes that are absent from all somatic cancer gene databases (Continued)

\begin{tabular}{|c|c|c|c|c|c|c|c|}
\hline & & & $\begin{array}{l}\text { Bile duct/ } \\
\text { gallbladder } \\
\text { cancer }\end{array}$ & & $\begin{array}{l}\text { Involved in step } 1 \text { of the subpathway } \\
\text { that synthesizes isocitrate from } \\
\text { oxaloacetate }\end{array}$ & $\begin{array}{l}\text { Well localized peaks of missense } \\
\text { mutations in the citrate synthase } \\
\text { domain }\end{array}$ & \\
\hline RGPD8 & $1760-1760$ & $2.200^{*} 10^{-6}$ & $\begin{array}{l}\text { Prostate cancer, } \\
\text { Glioma }\end{array}$ & $\begin{array}{l}\text { RANBP2-like and GRIP } \\
\text { domain-containing } \\
\text { protein } 8\end{array}$ & Unknown & $\begin{array}{l}\text { Single peak of missense mutations } \\
\text { at the C-terminal, possibly } \\
\text { disordered region }\end{array}$ & $\begin{array}{l}\text { Very pleriminaty indication } \\
\text { of possible marker role in } \\
\text { some cancer types }\end{array}$ \\
\hline KRTAP4-9 & $57-57$ & $3.407^{*} 10^{-6}$ & Breast cancer & $\begin{array}{l}\text { Keratin-associated } \\
\text { protein 4-9 }\end{array}$ & \multirow{3}{*}{$\begin{array}{l}\text { Part of an interfilamentous matrix, } \\
\text { in which hair keratin intermediate } \\
\text { filaments are embedded }\end{array}$} & Peak of missense mutations & \multirow{3}{*}{$\begin{array}{l}\text { Located in a potential breakpoint } \\
\text { initiating ERBB2 amplification, } \\
\text { which is known to be involved } \\
\text { in breast cancer (PMID: 23181561) }\end{array}$} \\
\hline KRTAP4-8 & $95-95$ & $5.261^{*} 10^{-6}$ & Glioma & $\begin{array}{l}\text { Keratin-associated } \\
\text { protein } 4-8\end{array}$ & & Peak of missense mutations & \\
\hline KRTAP9-9 & $18-30$ & $9.921^{*} 10^{-6}$ & $\begin{array}{l}\text { Pancreatic } \\
\text { cancer, Breast } \\
\text { cancer }\end{array}$ & $\begin{array}{l}\text { Keratin-associated } \\
\text { protein 9-9 }\end{array}$ & & $\begin{array}{l}\text { Short region dominated by } \\
\text { indels }\end{array}$ & \\
\hline
\end{tabular}


association between identified regions and functional protein regions, iSiMPRe may be useful to help in classifying mutations as drivers or passengers; simply based on their location inside or outside of a SiMPRe.

The fundamental differences between passenger and driver mutations can influence the set of preferential amino acid substitutions. In recent analyses, COSMIC mutations in the case of EGFR [51] and also for all genes [52] showed an uneven distribution of missense substitutions among cancer driver mutations defined as reoccurring mutation compared to likely passenger mutations recorded only once in COSMIC. Partitioning driver and passenger mutations based on iSiMPRe showed good agreement with preferential distributions observed earlier (see Additional file 2), with the seven and 16 most frequent substitutions appearing in both lists for driver and passenger mutations respectively. These results also hint at the partitioning power of iSiMPRe between driver and passenger missense mutations.

\section{The knowledge of significantly mutated regions involved in cancer can guide treatment and drug development choices}

One of the main advantages of the method proposed in this work is that it is able to identify not only cancer driver genes but also specific regions that are involved in the disease. The importance of this more detailed view is apparent in cases where a single protein has multiple regions that are specifically mutated in different cancer types. An example for this behavior is exhibited in the case of cytokine receptors (Fig. 5b). In these cases various forms of cancer target different protein regions, which implies a tissue-specific biological selection for particular mutations. Unfortunately, little is known about the mechanistic differences of the same receptors in different tissues that would explain the selection for non-overlapping mutational hotspots. One possible explanation could be the homo- or heterodimerization of the same receptor with different partners in different tissues. Facultative receptor tyrosine kinase heterodimerization is a common phenomenon and already documented for several related proteins [53-55].

The current collections of genes that have been causatively linked to tumor formation are heterogeneous concerning their dominant genetic alterations by which their structure/function may be modulated. An important question is how well genes containing SiMPRes (calculated from somatic missense mutations and short in-frame indels) represent all cancer genes. A surprising outcome of our analyses was that significantly mutated regions can be found in the majority of cancer genes, regardless of their dominant genetic alterations (Figs. 3 and 4). The two main exceptions are chromosomal translocations and rearrangements, which represent roughly independent modulations of the genome (Fig. 3). In many other cases, however, somatic mutations can have similar effects to other types of genetic alterations. (Note that the detailed analysis of non-coding intergenic, promoter or intronic mutations was not done, yet the data published by other groups hints that the targets of these alterations might also be somewhat separate from those targeted by somatic mutations [56-58].)

The observed interchangeability of various genetic alterations and the accumulation of somatic mutations is relatively straight-forward in the case where the dominant alterations diminish or abolish protein function (eg. deletion/loss, downregulation/underexpression and promoter hypermethylation). An example is provided by the SMAD4 gene (Fig. 6, top row). In this case the clustering of somatic mutations affects the C-terminal $\mathrm{MH} 2$ domain that is essential for both homo- and heterooligomerization. Normally SMAD4 transmits signals in the TGF-beta pathway, which is a negative regulator of epithelial growth. The deletion of the SMAD4 gene or the abolishment of the protein function via somatic mutations leads to cancer through the breaking of a negative regulatory pathway.

In the case of genetic alterations that enhance protein function, the interchangeability with missense mutations is slightly less evident. Overexpression/amplification typically can happen in receptors containing intracellular kinase domains that are able to modulate various pathways (such as ERK, JNK, Akt, etc.) via their downstream targets. Both amplification and their accumulated local somatic mutations force these proteins to be constitutively active independently from the binding of their extracellular ligands. For example in the case of EGFR (Fig. 6, middle row) the active form of the receptor is brought about by homo- or hetero-dimerization, which is normally achieved by the binding of EGF/TGFa (or other ligands). This dimerization can occur without ligand binding when the protein is overexpressed; however, the same effect can also be brought about by the accumulation of local somatic mutations in the $\mathrm{N}$-terminal part of the intracellular kinase domain.

As opposed to the previously discussed genetic alterations, in general the effects of translocations and chromosomal rearrangements cannot be understood through the function of a single gene/protein. The resulting fusion protein can carry functional parts from both original proteins and can fulfill novel biological roles. In most cases, the effect of translocations cannot be effectively mimicked by the accumulation of local mutations. However, in certain cases, genes undergoing translocations as their primary genetic alteration still show a significant accumulation of somatic mutations. A prime example for this behavior is presented by RUNX1 


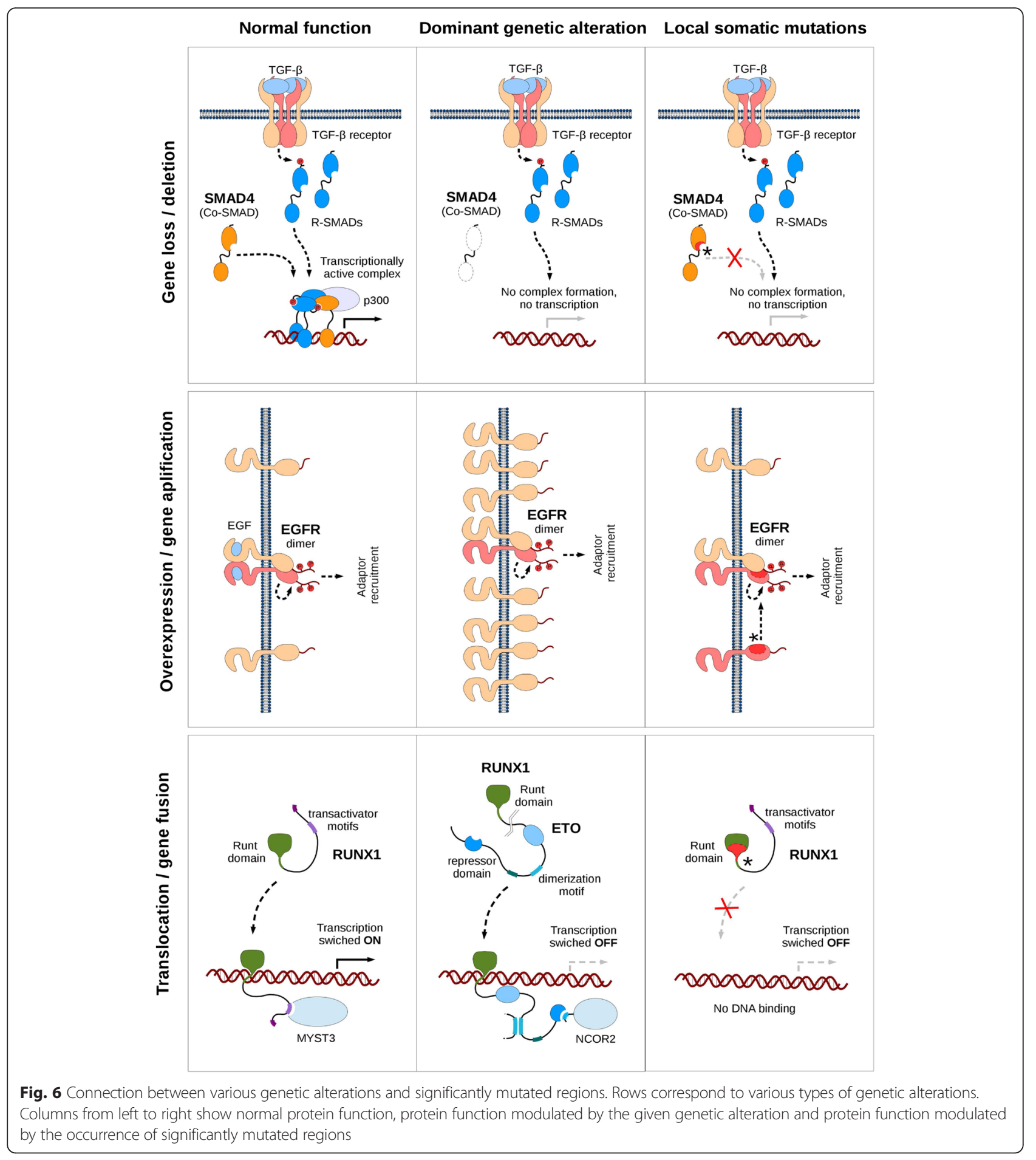

(Fig. 6, bottom row). This gene encodes a transcription factor that consists of the runt domain that is responsible for the interaction with the DNA and a long, presumably disordered transactivation region that binds to partner proteins. A common genetic alteration of RUNX1 is the fusion of the runt domain coding segment (first 5 exons) of the gene to the almost complete ETO gene. The resulting chimeric protein termed AML-ETO retains the ability to bind RUNX1 target genes while exerting the transcription repression function of ETO, silencing RUNX1 genes contributing to the development of various hematopoietic cancers, most notably AML. Apart from translocation, the RUNX1 gene also harbors significant accumulation of somatic mutations in the 
runt domain. The majority of these mutations were derived from AML samples indicating the possibility of these mutations mimicking the effect of the translocation. The mutation hotspots in the runt domain coincide with three regions that are directly involved in DNA binding mostly affecting arginine/lysine residues that are necessary for the charge complementarity. This diminishes the DNA binding capability of RUNX1 and inhibits the activation of its target genes, achieving the gene silencing effect of the gene fusion.

\section{Conclusions}

The observed somatic mutations collected across diverse tumor samples tend to cluster in functional protein regions that play a key role in tumorigenesis. This was exploited to construct an algorithm - iSiMPRe - which takes the list of the observed non-synomymous mutations and automatically identifies not only potential cancer driver genes, but also specific regions that are involved in the disease development. iSiMPRe not only outperforms other available methods, but its success also shows that the limited range of mutations considered (somatic exonic mutations that have a local effect) is enough to identify the majority of known cancer genes with a wide variety of typical genetic alterations (somatic gene deletions, gene amplifications, over- or underexpression, etc.). This way, the accumulation of somatic mutations can also offer potential sites for drug development efforts even in cases which are generally altered by more complex genetic mechanisms. Furthermore, most tumor suppressors also show local enrichments of missense mutations, just as proto-oncogens do. Nevertheless, a subset of cancer driver genes cannot be identified solely based on their enrichment of local somatic mutations. These typically involve genes dominantly altered by chromosomal translocations or rearrangements, or a subset of tumor suppressor genes that are altered mostly by truncating mutations. The complete recovery of cancer driver genes therefore requires a combined approach [39].

In the future, iSiMPRe could be a useful tool to identify genes and proteins where local biological selection affects their function in a selected way. This could not only be utilized to find (causatively) cancer-associated genes, but also to study their domains and other submolecular functional elements directly affected by mutations, even when no structural or functional annotation is available. Furthermore, apart from data supplied by cancer sequencing efforts, mutation data from any other illness targeted by systematic studies (Crohn's disease [59], autoimmunogenic disorders [60], autisms [61] and others $[62,63])$ can be used as input, thus iSiMPRe can be used to analyze the genetic background of rare hereditary diseases by linking phenotypic observations with protein function directly. The widely used approach of considering whole genes/proteins is problematic as these are often pleitropic, while for regions pathological involvement is more direct. This way iSiMPRe can be directly used to gain invaluable insight into the patomechanisms of diseases as well.

\section{Methods \\ COSMIC somatic mutations}

Cancer related somatic mutations were collected from version 73 of the COSMIC database (http://cancer. sanger.ac.uk/cosmic/) [8]. The complete dataset contained 20,337 protein sequences with at least one annotated mutation in the 73 version of the COSMIC database. All missense point mutations together with in-frame insertions and deletions were collected and checked against the protein sequences given in COSMIC. Mutations that were sequenced in samples having over 100 mutations were discarded as hypermutated samples, as these likely include a large fraction of passenger mutations. Furthermore, point mutations coinciding with positions of common single nucleotide polymorphisms were discarded using SNP data collected from the UCSC Genome Browser [64]. In order to filter out clonal variants, samples having at least five mutations and sharing at least $50 \%$ of the local variations (missense mutations and insertions and deletions) were filtered and only mutations coming from the most mutation-rich sample were considered to avoid the inclusion of duplicated entries. Altogether, this resulted in the inclusion of 404,913 missense mutations and 9977 in-frame insertions and deletions. Correctly defined mutations were linked to one of the cancer types based on their given sites of occurrence and histology according to the rules given in Additional file 2.

In the COSMIC database mutational data can be mapped to multiple isoforms. The isoform with the largest number of mutations often does not necessarily correspond to the canonic or the main isoform used in other databases. In this work, the isoforms of the same gene were clustered together and the isoform with the the highest number of mutations was selected. For functional and structural annotations and crosslinks to other databases, COSMIC gene ID's were also mapped to UniProt protein accession codes from the 2016/01 version of UniProt. The entries in each database and the identified regions were mapped to this main isoform, where possible.

\section{Databases of known cancer related genes}

In our study we surveyed four source databases that contained known cancer genes based on expert curation.

\section{COSMIC census genes}

Apart from mutations and larger scale genetic alterations, the COSMIC database also includes a list of 
census genes connected to cancer [65]. Somatically altered genes were collected and linked to one or more cancer types. This search yielded 824 gene-cancer type pairs.

\section{Driver genes}

A set of manually assembled cancer driver genes affected by somatic mutations was taken from [3]. This dataset also grouped genes as either tumor suppressors or oncogenes. The list included 54 oncogenes and 71 tumor suppressor genes.

\section{KEGG disease genes}

Genes connected to specific types of cancer were collected from the appropriate KEGG Disease pages [66]. For a wide variety of cancers, several genes are quoted together with their dominant type of genetic alterations. Genes are identified with HSA identifiers that are crosslinked with UniProt IDs. Based on this, genes were mapped to COSMIC sequences and the KEGG cancer types were assigned to one of the standardized cancer types providing a list of COSMIC genes with involvement in specific types of cancer. This search yielded 374 gene-cancer type pairs.

\section{OMIM cancer genes}

The Online Mendelian Inheritance in Man provides information about genes with disease association - including cancer [67]. These genes were accessed via UniProt (http://www.uniprot.org/docs/mimtosp) and mapped to COSMIC gene IDs. The referenced OMIM pages were parsed and manually curated. Those pages that described one or more types of cancer were annotated with one or more of the 27 standardized cancer types (see Table 2). This yielded a set of 979 gene-cancer type pairs. In this case, genes affected by somatic or germline mutations could not be generally discriminated.

\section{Somatic cancer gene dataset}

The number of genes contained in the four datasets containing somatic cancer genes (OMIM, COSMIC census, Drivers and KEGG), together with the amount of overlap between various datasets is shown in the figure in Additional file 4 . The fairly limited overlap among the datasets clearly indicates the lack of consensus on cancer genes. The total number of genes that are mentioned in all four datasets is only 32 , whereas there are 457 genes annotated as cancer-related that are present only in the OMIM database. In order to reduce the number of falsely annotated genes, only those were considered in the evaluation which occurred in at least two databases of the four. This resulted in 260 cancer genes (referred to as the SCGD (Somatic Cancer Gene Dataset); for an extensive list see table in Additional file 2).
Identification of significantly mutated protein regions A novel method was developed to identify significantly mutated protein regions. We refer this method as iSiMPRe (identification of significantly mutated protein regions) and the identified regions as 'SiMPRe's. iSiMPRe seeks to find regions that harbor a significantly enriched amount of somatic mutations compared to neutral local mutations which are assumed to be distributed evenly throughout the sequence. To discard a large portion of possible sequencing artefacts, iSiMPRe first filters out mutations that fall into genomic regions with low sequence complexity measured by TRF [68]. Next, the method follows a hierarchical, stepwise algorithm at the determination of possible significant regions, described in detail in Additional file 1 and in short in Fig. 1. Each identified region has a $p$-value assigned that characterizes the significance of the given region based on all three types of mutations. Based on this total $p$-value, regions are classified as high significance (if $p<10^{-20}$ ), medium significance $\left(10^{-20}<p<10^{-05}\right)$ or low significance $\left(10^{-05}<p<10^{-02}\right)$. Within that, however, the dominant mutation type (missense, insertion or deletion) is established for a region based on the relative contribution of each mutation type to the total significance of the region. Here the $p$-values of the found regions were re-calculated with only considering one class of the three possible mutations types. The mutation type yielding the lowest $p$-value on its own was considered to be the dominant one. Furthermore, the resulting regions were assigned to one or more of the 27 cancer types based on the histology and site annotations of the mutations present in the region. These cancer types are shown in Table 2.

\section{Comparison of iSiMPRe with oncodriveclust and edriver}

The performance of our method was compared to two methods with similar scope, OncoDriveClust [16] and eDriver [17]. For both methods the programs were installed and run locally with the default parameters. For the sake of direct comparison, all three methods were tested on the same set of mutations assembled from COSMIC.

\section{Association of functional regions with SiMPRes}

For each protein in the COSMIC dataset, functional and structural annotations were collected from the corresponding UniProt entry from the UniProt version 2016/01. Additionally, Pfam families, and known instances of linear motif sites collected in the ELM database were also mapped to the studied protein sequences. Altogether 18 categories were assigned to analyze the annotation of the significantly mutated regions (SiMPRe) including domains, motifs, repeats, kinases and enzymes (from Pfam [27]), DNA binding 
regions, regions of interest, transmembrane regions, nucleotide phosphate binding regions, active sites, binding sites and post-translational modification sites (from UniProt [26]), motifs, switches and phosphorylation sites (from ELM [28] and phosphoELM [69]). The possible over- and under-representation of these functional protein regions in SiMPRes was also determined and tested for statistical significance. For this, a random baseline was established. Each of the 534 found SiMPRe was moved to a randomly selected protein in a way that the length and disorder content of the original and the new, random protein had to be the same within $10 \%$ to avoid structural bias. Next, both real and randomly selected SiMPRes were assessed regarding their overlap with various functional protein units. The random region selection procedure was repeated 1000 times and the average and the standard deviation of the overlap between randomized regions and functional protein regions was calculated. The over-representation of SiMPRe in functional regions compared to randomized regions is given in standard error (StdErr) units in Table 3. As values calculated on random regions closely follow Gaussian distributions, the threshold for $p<0.01$ statistical significance in StdErr units is 2.326, therefore all color-coded overrepresentations are statistically significant.

\section{Assessment of protein disorder}

In the process of generating random regions, the disorder content of proteins were assessed using IUPred [70, 71], ANCHOR [72, 73] and Pfam [27]. The output of IUPred was smoothed in a window of 31 residues. Positions with a smoothed IUPred score over 0.5 or with an ANCHOR score over 0.5 were considered disordered, except for positions included in specific Pfam families which were annotated as domains.

\section{Substitution rates}

Substitution rates were calculated from missense mutations from the COSMIC database. Each type of missense mutation was considered only once for each position. First, all types of occurring missense mutations were collected separately for all positions. The number of substitutions changing the original amino acid of type $\mathrm{i}$ to type $\mathrm{j}$ is denoted by $n_{i-j}$ and substitution percentages are calculated as follows: $R_{i-j}=\left(n_{i-j} / N\right)^{*} 100$, where $\mathrm{N}$ is the total number of missense mutations. These substitution percentages were calculated separately for mutations inside and outside of SiMPRes and are shown in Additional file 2.

\section{Availability}

iSiMPRe is available for download from https:/github. com/BalintMeszaros/iSiMPRe.

\section{Reviewers' comments}

The authors would like to thank the reviewers for the careful reading of our manuscript and their constructive comments. The manuscript was revised in light of their statements. After each of the reviewers' comments, the authors address each of the comments in detail.

\section{Reviewer's report 1: Sándor Pongor, International Centre for Genetic Engineering and Biotechnology (ICGEB), Italy Reviewer comments}

Reviewer summary One of the key problems in cancer genomics data is the identification of driver mutations distinction from from passengers mutations. There are a number of known algorithms for the purpose but current approaches are often limited by biases in structural assignments as well as by the inaccuracy of the statistical background models. To stear clear of these problems the authors offer statistically-based method that considers statistically significant missense mutations, in-frame insertions and deletions in a unified statistical framework.

Reviewer recommendations to authors This is a piece of careful work and the results are convincing. One important issue is how an method can be maintained as the databases change and new raw data become available. the authors may want to add a few sentences how their method differs in this respect from other methods.

Author's response: iSiMPRe is constructed based on a unified statistical model and only a few empirical parameters are present in the method. Furthermore, these parameters (eg. the lengths of the seed regions used, which represent the typical sizes of functional protein regions) are independent of the databases used (eg. COSMIC) and thus the future variations of these databases are not expected to have a significant impact of the performance of iSiMPRe. However, the results, such as the list of potential cancer-related genes are heavily dependent on the input database, and accordingly we developed all our testing protocols to be easily updatable. This makes our workflow extremely capable of conducting follow-up analysis that show e.g. how new cancer genes emerge as a result of the accumulation of sequencing data. The opening paragraph of the Results section was updated to reflect this with the following sentences: "The input of iSiMPRe is a set of cancer-related missense mutations and in-frame insertions and deletions. The background mutation rate is calculated simply from these two files using only a few empirical parameters. This is in contrast to OncoDriveClust which estimates the background mutation rate from a set of silent substitutions that has to be supplied as a separate set of input data. iSiMPRe is described in detail in Additional file 1 (iSiMPRe 
protocol) and in short in Fig. 1. In order to enable potential users to apply the method to updated versions of COSMIC datasets or other sources of cancer mutation data, the source code of iSiMPRe is available for download. In our experience, the identified significantly mutated regions change very little with updates of COSMIC datasets."

\section{Minor issues}

The authors may want to go through the English of text which is however clear and understandable in its present form.

Author's response: We have made extra care to correct the English of the text.

\section{Reviewer's report 2: Michael Gromiha, Indian Institute of Technology Madras, India \\ Reviewer comments}

Reviewer summary In this work, the authors presented a novel method for identifying regions that are significantly enriched in somatic mutations and indels. The analysis on human proteome showed the presence of about around 500 protein regions linked with 27 distinct cancer types. It also identified novel genes and regions that have not yet been associated with cancer. The area of research is interesting and potential applications to cancer research. The manuscript is well written and analysis has been made in details.

Reviewer recommendations to authors Following points may be considered for improvements and discussions.

1. Recently, preferred amino acid mutations in cancer genes have been reported using COSMIC database with the location of mutations. A comparison of those results with the present work could be useful.

Author's response: The substitution rates calculated for the missense mutations inside and outside SiMPRes have been contrasted with the mutation rates detailed in a recent study by Gomiha et al. (Exploring prefered amino acid mutations in cancer genes, applications to identify potential drug targets). Although the two studies use different definitions for driver and passenger mutations (being present in COSMIC multiple times vs being inside a significantly mutated region), the two distributions of preferred amino acid changes show a striking similarity for both drivers and passengers mutations. The cited analysis found 26 driver amino acid substitutions (out of the possible 380) that represent over $1 \%$ of total observed substitutions each. Out of these, the top 7 ones are present in our analysis as well, with 9 more appearing with over $1 \%$ frequency. For the passenger mutations, Gromiha et al. described 29 mutations with over $1 \%$ occurrence, out of which the top 16 (and an additional 10) are present in our list of frequent substitutions as well. The results of the analysis have been included in the supplementary data and have been referred in the discussion section: "The fundamental differences between passenger and driver mutations can influence the set of preferential amino acid substitutions. In recent analyses, COSMIC mutations in the case of EGFR and also for all genes showed an uneven distribution of missense substitutions among cancer driver mutations defined as reoccurring mutation compared to likely passenger mutations recorded only once in COSMIC. Partitioning driver and passenger mutations based on iSiMPRe showed good agreement with preferential distributions observed earlier (see Additional file 2), with the 7 and 16 most frequent substitutions appearing in both lists for driver and passenger mutations respectively. These results also hint at the partitioning power of iSiMPRe between driver and passenger missense mutations."

2. The preferred localized regions may be discussed in terms of secondary structures and solvent accessibility (either experimental or predicted).

Author's response: While secondary structure and solvent accessibility properties can be informative for globular proteins, they cannot be directly applied to intrinsically disordered proteins (IDPs). Since a significant portion of the mutated regions can correspond to IDPs, we believe that without partitioning our results based on available structural data and analyzing IDPs and structured protein regions separately, the structural bias would make results difficult to interpret properly. We believe that this structure-based analysis would be really interesting but we also believe that this work is outside the scope of the present paper and will be presented in future publications.

3. COSMIC database provides the counts for the mutants. The number of counts used to define somatic mutations may be mentioned.

Author's response: These data were added to both the 'In-frame indels are important for finding cancer genes' section of Results and the Methods section.

\section{Reviewer's report 3: Zoltán Gáspári, Pázmány University, Budapest \\ Reviewer comments}

Reviewer summary The manuscript addresses an important problem within a timely topic, namely, the identification of mutational hotspots with biological significance within proteins involved in various forms of cancer. The study is carefully designed and the presentation of the main points of the methodoology and results is generally clear. The iSiMPRE program developed is freely available and the source code is clearly readable. The results provide important novel insights 
into the role of missense mutations and indels in various proteins and their regions. They can form the basis of further studies on the possible roles of the proteins and functional regions identified in the study.

Reviewer recommendations to authors The field of identifying driver mutations is highly complex. Despite some commonly accepted paradigms there are divergent approaches based on different considerations for the identification of driver genes and/or mutations with biological/therapeutic significance. Therefore, I think that the manuscript could benefit from a theoretical introduction about driver genes and their identification. In particular, it should be important to detail the premises on which the iSiMPRe method is expected to identify driver mutations more efficiently than other methods. This then can be referred in the subsection "The search for cancer driver genes continues" and provide the reader a firm background for the concept of the study. The conclusion section contains some hints, but I suggest that this should be described in a more detailed way in an earlier, suitable part of the paper.

Author's response: A separate paragraph has been included in the Discussion section to better shed light on the various approaches are used to identify cancer drives genes and the advantages iSiMPRe approach of this work might have over other methods: "In addition to manually curated databases, various computational methods have also been developed to identify cancer driver genes. Most commonly used approaches seek to identify cancer driver genes either in the context of pathways and protein interaction networks or by detecting signs of positive selection at the level of genes. Such methods can be based on the increased number of observed mutations compared to the background mutation rate; a high rate of non-silent mutations compared to silent mutations; or on the bias towards the accumulation of mutations with high functional impact. However, the accumulation of mutations can highlight not only genes but also specific functional regions at the protein level that are involved in disease development. Recent methods, such as OncoDriveClust or e-Driver identify putative cancer driver genes based on this concept, similarly to the iSiMPre method presented in this work. Clustering of mutations can also be observed in three-dimensional protein structures that often correspond to perturbed protein-protein interaction sites. Given the complexity and heterogeneity of the molecular basis of cancer, the combination of different signals of positive selection can more reliably indicate mutational drivers. Nevertheless, in our experience, the iSiMPre method is able to identify the majority of cancer driver genes based on the clustering of mutations and outperforms methods with similar scope. The increased performance of iSiMPre can be attributed to several factors, including the cleaning of mutational data (e.g. eliminating likely neutral polymorphisms and mutations occurring within tandem repeats that are more likely to accommodate neutral mutations as well as sequencing errors). Additional factor is the incorporation of all genetic variations with positional information, which include short in-frame insertions and deletions while excluding frameshift and non-sense mutations. iSiMPre is based on an unbiased approach that does not rely on previous knowledge of structure or domain, which could be especially important to detect cancer driver mutations located in intrinsically disordered proteins for example (manuscript in preparation)."

Major recomenndations: In general, the study is welldocumented, although there are some details in the methodology that, in my opinion, need to be clarified.

- How were disordered regions identified and how was the extent of disorder assessed for use in the randomization process?

Author's response: This has been added to the Methods chapter in a separate section.

- Were the categories 'Kinase,, 'Enzyme,' 'Domain' used in a mutually exclusive way?

Author's response: No, they are used in accordance with standard Pfam definitions, meaning that all kinases are a subset of enzymes which are in turn a subset of domains.

How exactly were the 'regions of interest' defined (which UniProt keywords were included here)?

Author's response: UniProt contains a 'regions of interest' keyword, covering a broad range of functional protein regions. The appropriate section of Results was updated to reflect this.

Which version of UniProt was used?

Author's response: 2016/01 version, this information has been added to the Methods section as well.

- In all studies using "artificial" thresholds, it could be important to justify the choice of these. Do the authors have data on how inclusion of hypermutated sequences containing more than 100 mutations affect their conclusions? How can the significance regions be justified?

Author's response: Although no systematic study was done, we calculated the main results with various cutoffs regarding allowed mutations counts per sample. While cutoffs lower than 100 produced apparently poorer results (several known cancer genes were lost from identification), cutoffs between 100 and 150 all yielded approximately the same results. The inclusion of samples with over 150 mutations slowly started to increase the number of identified low significance SiMPRes, however in a cancer type specific way (new regions appeared mainly conjunction with colorectal and lung cancers and melanoma). This indicated that data represented by samples with over 150 mutations contain significantly larger 
noise. As the region between 100 and 150 seemed to make no difference, we opted to exclude those data as well and used the cutoff of 100 mutations/sample in all final analyses.

Questions/recommendations with respect to the results: -The authors used the isoform with the most mutations. Could the validity of this approach checked at least for the top SiMPRes identified? Do the results obtained for the isoform chosen conform to those that could be obtained for the other isoforms, taking into account their specific role/localization etc.? -

Author's response: We fully agree that it would be very interesting to study significantly mutated regions in an isoform specific way. However, the current policy of the COSMIC database does not make is possible, as mutations in COSMIC are usually mapped to only one of the isoforms which is often as not the same as the primary isoform.

There is only limited information provided on affected domain types ("Within structured domains, there is a clear tendency for enzymes, especially for kinases, confirming earlier observations"). As the data is at hand, could a more detailed analysis provide some further insights?

Author's response: We now provide a more detailed table about various structural and functional annotations corresponding to SiMPRes and also added a sentence about the most commonly mutated domains:

"The most commonly mutated domain is the Tyrosine kinase domain observed in 20 different proteins, including such well-known cancer driver genes as ALK, EGFR or BRAF (see Additional file 3). Other domains mutated in multiple cases include the Serine/threonine-protein kinase domain (e.g. in Activin receptor type-1 or in Serine/threonine-protein kinase STK11) and the SH2 domains (e.g. in PIK3-R1 and STAT3)."

Minor questions/recommendations: -

Are there any selected cases where the authors can, with a detailed analysis of the literature, justify that most of the mutations themselves in a SiMPRe indeed significantly affect protein function? - It would be really interesting to analyze in detail one or two cases where different regions of the same protein are associated with different cancer types, but I understand if this is outside of the scope of the present study.

Author's response: We discuss several examples in the manuscript in some detail, including DNMT3A, PIK3R1, c-KIT and FGFR3, as well as SMAD4, EGFR and RUNX1 in the discussion. We would also like to emphasize that while most of highly significant mutated regions are well studied examples, for which we have relatively good understanding about the mechanism of the mutation, our understanding for many other cases is still limited. Therefore, we agree with the referee that analyses of further examples are outside of the scope of the present study.

\section{Minor issues}

Kindly be more specific in statements like "Nevertheless the majority of tumor suppressor genes (50 out of $71,70 \%$ )" as it is bit confusing to (seemingly?) compare numbers to percentages.

Author's response: These sentences were rephrased to avoid confusion.

I personally would refrain from using terms like "known oncogene" and would prefer "has been described as an oncogene" or similar.

Author's response: These sentences were re-written to exclude these expressions.

In the sentence "The current collections of genes that have been casually linked to tumor formation" I guess the authors meant "causatively"? Please corrent "genominc" here: "genominc regions with low sequence complexity measured by TRF [62]"

Author's response: These were corrected.

In Table 4, please add all relevant references weher applicable (e.g. I guess where "Some indication" has been described, there is some kind of reference).

Author's response: The reference has been added.

Please re-read Additional file 1 and correct typos/small grammatical errors.

Author's response: We made extra effort to correct typos and small grammatical errors in the Additional file 1.

I suggest to merge all additinal xls files into a single file with multiple tabs.

Author's response: All additional tables were merged into a single file.

\section{Additional files}

Additional file 1: iSiMPRe protocol. (PDF $415 \mathrm{~kb}$ )

Additional file 2: All supplementary tables merged. 1: List of all identified SiMPRes. 2: All novel genes that contain identified low significance SiMPRes. 3: Substitution percentages calculated for mutations in and outside of SiMPRes. 4: Criteria for the classification of somatic mutations. 5: The elements of the SCGD dataset with support information. (XLS $285 \mathrm{~kb}$ )

Additional file 3: iSiMPRe mini-website. The mini-website shows all identified SiMPRes together with the found annotations from various source databases (Pfam, UniProt, ELM, etc.). (ZIP $37 \mathrm{~kb}$ )

Additional file 4: Cancer driver gene datasets. (PNG $131 \mathrm{~kb}$ )

\section{Abbreviations}

AML: acute myeloid leukemia; CHEK2: CHEckpoint kinase 2; CML: chronic myeloid leukemia; COSMIC: catalogue of somatic mutations in cancer; DNMT3A: DNA methyltransferase 3A; EGFR: epidermal growth factor receptor; FGFR3: fibroblast growth factor receptor 3; GIST: gastrointestinal stromal tumor; IL7R: Interleukin-7 receptor; iSiMPRe: identification of Significantly Mutated Protein Regions; KEGG: kyoto encyclopedia of genes and genomes; LOH: loss of heterozygosity; OMIM: online mendelian inheritance in man; PI3K-R1: phosphatidylinositol 3-kinase regulatory subunit alpha; PRC2: polycomb repressive complex 2; SCGD: somatic cancer gene 
dataset; SiMPRe: significantly mutated protein regions; SNV: single nucleotide variation; STAT3: signal transducer and activator of transcription 3.

\section{Competing interests}

The authors declare that they have no competing interests.

\section{Authors' contributions}

BM carried out the database assembly, performed the calculations, conceived the construction of iSiMPRe and drafted the manuscript. AZ participated in figure preparation and helped to draft the manuscript. AR coordinated the biological aspects of the study design and revised the manuscript. IS helped to discuss the results and to draft the manuscript. ZD conceived the study and participated in its design and coordination and helped to draft the manuscript. All authors read and approved the final manuscript.

\section{Acknowledgements}

The authors wish to thank Borbála Hajdu-Soltész for her corrections on the manuscript.

\section{Funding}

This work was supported by the Hungarian Scientific Research Fund (grant number: OTKA K 108798, NK 100482, K 115698 and OTKA NN 114309); A.R. and Z. D are the recipients of the "Lendület" Grant from the Hungarian Academy of Sciences (LP2013-57, and LP2014-18, respectively).

\section{Author details}

${ }^{1}$ Institute of Enzymology, Research Centre for Natural Sciences, Hungarian Academy of Sciences, 2 Magyar Tudósok krt, Budapest H-1117, Hungary. ${ }^{2}$ Lendület Protein Interaction Group, Institute of Enzymology, Research Centre for Natural Sciences, Hungarian Academy of Sciences, 2 Magyar Tudósok krt, Budapest H-1117, Hungary. ${ }^{3}$ MTA-ELTE Lendület Bioinformatics Research Group, Department of Biochemistry, Eötvös Loránd University, 11/c Pázmány Péter stny, Budapest H-1117, Hungary.

\section{Received: 11 February 2016 Accepted: 20 April 2016}

\section{Published online: 05 May 2016}

\section{References}

1. Wood LD, Parsons DW, Jones S, Lin J, Sjoblom T, Leary RJ, Shen D, Boca SM, Barber T, Ptak J, et al. The genomic landscapes of human breast and colorectal cancers. Science. 2007:318:1108-13.

2. Greenman C, Stephens P, Smith R, Dalgliesh GL, Hunter C, Bignell G, Davies $H$, Teague J, Butler A, Stevens C, et al. Patterns of somatic mutation in human cancer genomes. Nature. 2007:446:153-8

3. Vogelstein B, Papadopoulos N, Velculescu VE, Zhou S, Diaz Jr LA, Kinzler KW. Cancer genome landscapes. Science. 2013;339:1546-58.

4. Stratton MR, Campbell PJ, Futreal PA. The cancer genome. Nature. 2009:458: 719-24.

5. Comprehensive genomic characterization defines human glioblastoma genes and core pathways. Nature 2008, 455:1061-1068.

6. Watson IR, Takahashi K, Futreal PA, Chin L. Emerging patterns of somatic mutations in cancer. Nat Rev Genet. 2013:14:703-18.

7. Pleasance ED, Cheetham RK, Stephens PJ, McBride DJ, Humphray SJ, Greenman CD, Varela I, Lin ML, Ordonez GR, Bignell GR, et al. A comprehensive catalogue of somatic mutations from a human cancer genome. Nature. 2010;463:191-6.

8. Forbes SA, Beare D, Gunasekaran P, Leung K, Bindal N, Boutselakis H, Ding M, Bamford S, Cole C, Ward S, et al. COSMIC: exploring the world's knowledge of somatic mutations in human cancer. Nucleic Acids Res. 2015: 43:D805-811.

9. Gonzalez-Perez A, Mustonen V, Reva B, Ritchie GR, Creixell P, Karchin R, Vazquez M, Fink JL, Kassahn KS, Pearson JV, et al. Computational approaches to identify functional genetic variants in cancer genomes. Nat Methods. 2013:10:723-9.

10. Lawrence MS, Stojanov P, Polak P, Kryukov GV, Cibulskis K, Sivachenko A, Carter SL, Stewart C, Mermel CH, Roberts SA, et al. Mutational heterogeneity in cancer and the search for new cancer-associated genes. Nature. 2013;499: 214-8.

11. Bignell GR, Greenman CD, Davies H, Butler AP, Edkins S, Andrews JM, Buck $G$, Chen L, Beare D, Latimer C, et al. Signatures of mutation and selection in the cancer genome. Nature. 2010;463:893-8.
12. Gibson TJ. Cell regulation: determined to signal discrete cooperation. Trends Biochem Sci. 2009:34:471-82.

13. van der Lee R, Buljan M, Lang B, Weatheritt RJ, Daughdrill GW, Dunker AK, Fuxreiter M, Gough J, Gsponer J, Jones DT, et al. Classification of intrinsically disordered regions and proteins. Chem Rev. 2014;114:6589-631.

14. Joerger AC, Fersht AR. Structure-function-rescue: the diverse nature of common p53 cancer mutants. Oncogene. 2007;26:2226-42.

15. Yang F, Petsalaki E, Rolland T, Hill DE, Vidal M, Roth FP. Protein domain-level landscape of cancer-type-specific somatic mutations. PLoS Comput Biol. 2015:11:e1004147.

16. Tamborero D, Gonzalez-Perez A, Lopez-Bigas N. OncodriveCLUST: exploiting the positional clustering of somatic mutations to identify cancer genes. Bioinformatics. 2013:29:2238-44.

17. Porta-Pardo E, Godzik A. e-Driver: a novel method to identify protein regions driving cancer. Bioinformatics. 2014;30(3):109-3114.

18. Porta-Pardo E, Hrabe T, Godzik A. Cancer3D: understanding cancer mutations through protein structures. Nucleic Acids Res. 2015;43:D968-973.

19. Kamburov A, Lawrence MS, Polak P, Leshchiner I, Lage K, Golub TR, Lander ES, Getz G. Comprehensive assessment of cancer missense mutation clustering in protein structures. Proc Natl Acad Sci U S A. 2015;112:E5486-5495.

20. Nehrt NL, Peterson TA, Park D, Kann MG. Domain landscapes of somatic mutations in cancer. BMC Genomics. 2012;13(4):S9.

21. Kanai $Y$, Ushijima S, Nakanishi $Y$, Sakamoto M, Hirohashi S. Mutation of the DNA methyltransferase (DNMT) 1 gene in human colorectal cancers. Cancer Lett. 2003;192:75-82

22. Bozic I, Antal T, Ohtsuki H, Carter H, Kim D, Chen S, Karchin R, Kinzler KW, Vogelstein B, Nowak MA. Accumulation of driver and passenger mutations during tumor progression. Proc Natl Acad Sci U S A. 2010;107:18545-50.

23. Kim MS, Chung NG, Yoo NJ, Lee SH. Somatic mutation of IL7R exon 6 in acute leukemias and solid cancers. Hum Pathol. 2013:44:551-5.

24. Li S, Thangapazham RL, Wang JA, Rajesh S, Kao TC, Sperling L, Moss J, Darling TN. Human TSC2-null fibroblast-like cells induce hair follicle neogenesis and hamartoma morphogenesis. Nat Commun. 2011;2:235

25. Bell DW, Sikdar N, Lee KY, Price JC, Chatterjee R, Park HD, Fox J, Ishiai M, Rudd ML, Pollock LM, et al. Predisposition to cancer caused by genetic and functional defects of mammalian Atad5. PLoS Genet. 2011;7:e1002245.

26. UniProt: a hub for protein information. Nucleic Acids Res. 2015;43:D204-212.

27. Finn RD, Bateman A, Clements J, Coggill P, Eberhardt RY, Eddy SR, Heger A, Hetherington K, Holm L, Mistry J, et al. Pfam: the protein families database. Nucleic Acids Res. 2014:42:D222-230.

28. Dinkel H, Van Roey K, Michael S, Davey NE, Weatheritt RJ, Born D, Speck T, Kruger D, Grebnev G, Kuban M, et al. The eukaryotic linear motif resource ELM: 10 years and counting. Nucleic Acids Res. 2014;42:D259-266.

29. Pajkos M, Meszaros B, Simon I, Dosztanyi Z. Is there a biological cost of protein disorder? Analysis of cancer-associated mutations. Mol BioSyst. 2012 8:296-307.

30. Dixit A, Verkhivker GM. Structure-functional prediction and analysis of cancer mutation effects in protein kinases. Comput Math Methods Med. 2014;2014: 653487

31. Dixit A, Yi L, Gowthaman R, Torkamani A, Schork NJ, Verkhivker GM. Sequence and structure signatures of cancer mutation hotspots in protein kinases. PLoS One. 2009;4:e7485

32. Uyar B, Weatheritt RJ, Dinkel H, Davey NE, Gibson TJ. Proteome-wide analysis of human disease mutations in short linear motifs: neglected players in cancer? Mol BioSyst. 2014;10:2626-42.

33. Reimand J, Wagih O, Bader GD. The mutational landscape of phosphorylation signaling in cancer. Scientific reports. 2013;3:2651.

34. Lawrence MS, Stojanov P, Mermel CH, Robinson JT, Garraway LA, Golub TR, Meyerson M, Gabriel SB, Lander ES, Getz G. Discovery and saturation analysis of cancer genes across 21 tumour types. Nature. 2014;505:495-501.

35. Ding L, Wendl MC, McMichael JF, Raphael BJ. Expanding the computational toolbox for mining cancer genomes. Nat Rev Genet. 2014;15:556-70.

36. Tamborero D, Gonzalez-Perez A, Perez-Llamas C, Deu-Pons J, Kandoth C, Reimand J, Lawrence MS, Getz G, Bader GD, Ding L, Lopez-Bigas N. Comprehensive identification of mutational cancer driver genes across 12 tumor types. Scientific reports. 2013;3:2650.

37. Wendl MC, Wallis JW, Lin L, Kandoth C, Mardis ER, Wilson RK, Ding L. PathScan: a tool for discerning mutational significance in groups of putative cancer genes. Bioinformatics. 2011;27:1595-602.

38. Gonzalez-Perez A, Lopez-Bigas N. Functional impact bias reveals cancer drivers. Nucleic Acids Res. 2012;40:e169. 
39. Gonzalez-Perez A, Perez-Llamas C, Deu-Pons J, Tamborero D, Schroeder MP, Jene-Sanz A, Santos A, Lopez-Bigas N. IntOGen-mutations identifies cancer drivers across tumor types. Nat Methods. 2013;10:1081-2.

40. Dutta RK, Welander J, Brauckhoff M, Walz M, Alesina P, Arnesen T, Soderkvist P, Gimm O. Complementary somatic mutations of KCNJ5, ATP1A1, and ATP2B3 in sporadic aldosterone producing adrenal adenomas. Endocrinerelated cancer. 2014;21:L1-4.

41. Kraan W, Horlings HM, van Keimpema M, Schilder-Tol EJ, Oud ME, Scheepstra C, Kluin PM, Kersten MJ, Spaargaren M, Pals ST. High prevalence of oncogenic MYD88 and CD79B mutations in diffuse large B-cell lymphomas presenting at immune-privileged sites. Blood Cancer J. 2013;3:e139.

42. Robinson DR, Wu YM, Vats P, Su F, Lonigro RJ, Cao X, Kalyana-Sundaram S, Wang $R$, Ning $Y$, Hodges $L$, et al. Activating ESR1 mutations in hormoneresistant metastatic breast cancer. Nat Genet. 2013;45:1446-51.

43. Kakiuchi M, Nishizawa T, Ueda H, Gotoh K, Tanaka A, Hayashi A, Yamamoto S, Tatsuno K, Katoh H, Watanabe Y, et al. Recurrent gain-of-function mutations of RHOA in diffuse-type gastric carcinoma. Nat Genet. 2014;46:583-7.

44. Maxson JE, Gotlib J, Pollyea DA, Fleischman AG, Agarwal A, Eide CA, Bottomly D, Wilmot B, McWeeney SK, Tognon CE, et al. Oncogenic CSF3R mutations in chronic neutrophilic leukemia and atypical CML. N Engl J Med. 2013;368:1781-90.

45. Nagarajan N, Bertrand D, Hillmer AM, Zang ZJ, Yao F, Jacques PE, Teo AS, Cutcutache I, Zhang Z, Lee WH, et al. Whole-genome reconstruction and mutational signatures in gastric cancer. Genome Biol. 2012;13:R115.

46. Leir SH, Harris A. MUC6 mucin expression inhibits tumor cell invasion. Exp Cell Res. 2011;317:2408-19.

47. de Bolos C, Guma M, Barranco C, Garrido M, Kim YS, Real FX. MUC6 expression in breast tissues and cultured cells: abnormal expression in tumors and regulation by steroid hormones. Intern J Cancer J Intern du Cancer. 1998:77:193-9.

48. Kim N, Hong Y, Kwon D, Yoon S. Somatic mutaome profile in human cancer tissues. Genome Inform. 2013;11:239-44.

49. Lee KY, Fu H, Aladjem MI, Myung K. ATAD5 regulates the lifespan of DNA replication factories by modulating PCNA level on the chromatin. J Cell Biol. 2013;200:31-44.

50. Pilati C, Letouze E, Nault JC, Imbeaud S, Boulai A, Calderaro J, Poussin K, Franconi A, Couchy G, Morcrette G, et al. Genomic profiling of hepatocellular adenomas reveals recurrent FRK-activating mutations and the mechanisms of malignant transformation. Cancer Cell. 2014;25:428-41.

51. Anoosha P, Huang LT, Sakthivel R, Karunagaran D, Gromiha MM. Discrimination of driver and passenger mutations in epidermal growth factor receptor in cancer. Mutat Res. 2015;780:24-34.

52. Anoosha P, Sakthivel R, Michael Gromiha M. Exploring preferred amino acid mutations in cancer genes: Applications to identify potential drug targets. Biochim Biophys Acta. 1862;2016:155-65.

53. Faraone D, Aguzzi MS, Ragone G, Russo K, Capogrossi MC, Facchiano A. Heterodimerization of FGF-receptor 1 and PDGF-receptor-alpha: a novel mechanism underlying the inhibitory effect of PDGF-BB on FGF-2 in human cells. Blood. 2006;107:1896-902.

54. Citri A, Skaria KB, Yarden Y. The deaf and the dumb: the biology of ErbB-2 and ErbB-3. Exp Cell Res. 2003;284:54-65.

55. Cudmore MJ, Hewett PW, Ahmad S, Wang KQ, Cai M, Al-Ani B, Fujisawa T, Ma B, Sissaoui S, Ramma W, et al. The role of heterodimerization between VEGFR-1 and VEGFR-2 in the regulation of endothelial cell homeostasis. Nat Commun. 2012;3:972.

56. Tian E, Borset M, Sawyer JR, Brede G, Vatsveen TK, Hov H, Waage A, Barlogie B, Shaughnessy JD, Jr., Epstein J, Sundan A. Allelic mutations in noncoding genomic sequences construct novel transcription factor binding sites that promote gene overexpression. Genes Chromosomes Cancer. 2015;54:692-701.

57. Fredriksson NJ, Ny L, Nilsson JA, Larsson E. Systematic analysis of noncoding somatic mutations and gene expression alterations across 14 tumor types. Nat Genet. 2014;46:1258-63.

58. Weinhold N, Jacobsen A, Schultz N, Sander C, Lee W. Genome-wide analysis of noncoding regulatory mutations in cancer. Nat Genet. 2014;46:1160-5.

59. Petersen BS, Spehlmann ME, Raedler A, Stade B, Thomsen I, Rabionet R, Rosenstiel P, Schreiber S, Franke A. Whole genome and exome sequencing of monozygotic twins discordant for Crohn's disease. BMC Genomics. 2014; 15:564.

60. Hunt KA, Mistry V, Bockett NA, Ahmad T, Ban M, Barker JN, Barrett JC, Blackburn $\mathrm{H}$, Brand $\mathrm{O}$, Burren $\mathrm{O}$, et al. Negligible impact of rare autoimmune-locus coding-region variants on missing heritability. Nature. 2013;498:232-5.
61. Yuen RK, Thiruvahindrapuram B, Merico D, Walker S, Tammimies K, Hoang N, Chrysler C, Nalpathamkalam T, Pellecchia G, Liu Y, et al. Whole-genome sequencing of quartet families with autism spectrum disorder. Nat Med. 2015:21:185-91.

62. Yang Y, Muzny DM, Reid JG, Bainbridge MN, Willis A, Ward PA, Braxton A, Beuten J, Xia F, Niu Z, et al. Clinical whole-exome sequencing for the diagnosis of mendelian disorders. N Engl J Med. 2013;369:1502-11.

63. The UK10K project identifies rare variants in health and disease. Nature. 2015.

64. Karolchik D, Barber GP, Casper J, Clawson H, Cline MS, Diekhans M, Dreszer TR, Fujita PA, Guruvadoo L, Haeussler M, et al. The UCSC Genome Browser database: 2014 update. Nucleic Acids Res. 2014;42:D764-770.

65. Futreal PA, Coin L, Marshall M, Down T, Hubbard T, Wooster R, Rahman N, Stratton MR. A census of human cancer genes. Nat Rev Cancer. 2004;4:177-83.

66. Kanehisa M, Goto S, Furumichi M, Tanabe M, Hirakawa M. KEGG for representation and analysis of molecular networks involving diseases and drugs. Nucleic Acids Res. 2010;38:D355-360.

67. Hamosh A, Scott AF, Amberger JS, Bocchini CA, McKusick VA. Online Mendelian Inheritance in Man (OMIM), a knowledgebase of human genes and genetic disorders. Nucleic Acids Res. 2005;33:D514-517.

68. Benson G. Tandem repeats finder: a program to analyze DNA sequences. Nucleic Acids Res. 1999;27:573-80.

69. Dinkel H, Chica C, Via A, Gould CM, Jensen LJ, Gibson TJ, Diella F. Phospho. ELM: a database of phosphorylation sites-update 2011. Nucleic Acids Res. 2011;39:D261-267.

70. Dosztanyi Z, Csizmok V, Tompa P, Simon I. The pairwise energy content estimated from amino acid composition discriminates between folded and intrinsically unstructured proteins. J Mol Biol. 2005;347:827-39.

71. Dosztanyi Z, Csizmok V, Tompa P, Simon I. IUPred: web server for the prediction of intrinsically unstructured regions of proteins based on estimated energy content. Bioinformatics. 2005;21:3433-4.

72. Dosztanyi Z, Meszaros B, Simon I. ANCHOR: web server for predicting protein binding regions in disordered proteins. Bioinformatics. 2009;25: 2745-6.

73. Meszaros B, Simon I, Dosztanyi Z. Prediction of protein binding regions in disordered proteins. PLoS Comput Biol. 2009:5:e1000376.

\section{Submit your next manuscript to BioMed Central and we will help you at every step:}

- We accept pre-submission inquiries

- Our selector tool helps you to find the most relevant journal

- We provide round the clock customer support

- Convenient online submission

- Thorough peer review

- Inclusion in PubMed and all major indexing services

- Maximum visibility for your research

Submit your manuscript at www.biomedcentral.com/submit
BioMed Central 